\title{
Forecasting A Long Memory Process Subject to Structural Breaks
}

\author{
Cindy Shin-Huei Wang ${ }^{\dagger}$ Luc Bauwens ${ }^{\dagger \dagger}$ Cheng Hsiao $\dagger^{\dagger} \dagger$ \\ Jan 19, 2013
}

\begin{abstract}
We develop an easy-to-implement method for forecasting a stationary autoregressive fractionally integrated moving average (ARFIMA) process subject to structural breaks with unknown break dates. We show that an ARFIMA process subject to a mean shift and a change in the long memory parameter can be well approximated by an autoregressive (AR) model and suggest using an information criterion (AIC or Mallows' $C_{p}$ ) to choose the order of the approximate AR model. Our method avoids the issue of estimation inaccuracy of the long memory parameter and the issue of spurious breaks in finite sample. Insights from our theoretical analysis are confirmed by Monte Carlo experiments, through which we also find that our method provides a substantial improvement over existing prediction methods. An empirical application to the realized volatility of three exchange rates illustrates the usefulness of our forecasting procedure. The empirical success of the HAR-RV model is explained, from an econometric perspective, by our theoretical and simulation results.
\end{abstract}

Keywords: Forecasting, Long memory process, Structural break, HAR model.

JEL Classification: C22, C53.

\section{Introduction}

Macroeconomic and financial time series are subject to occasional structural breaks (see Stock and Watson, 1996 and Pesaran and Timmermann, 2005). It is often argued that ignoring the presence of breaks can lead to seriously biased estimates and forecasts (see Clements and Hendry, 1998). Accordingly, a conventional approach to forecast a time series with breaks is first to determine when the most

$\dagger$ Université catholique de Louvain, CORE, B-1348 Louvain-La-Neuve, Belgium and National TsingHwa University, Department of Quantitative Finance, Taiwan.

†† Université catholique de Louvain, CORE, B-1348 Louvain-La-Neuve, Belgium

††† University of Southern California, U.S.A., City University of Hong Kong and WISE, Xiamen University, China.

††† Corresponding author, E-Mail: chsiao@usc.edu 
recent break occurred and then to use the post-break data to estimate the forecasting model. Nevertheless, Pesaran and Timmermann (2005, 2007) showed that such an approach does not necessarily yield the optimal forecasting performance, especially when the time series are subject to multiple breaks, due to the difficulty in estimating the timing of breaks. Moreover, they illustrate that pre-break data can be useful for forecasting the after-break outcomes, provided that the break is not too large.

Many researchers use the autoregressive fractionally integrated moving average process of order $p, d, q$, denoted as $\operatorname{ARFIMA}(p, d, q)$, or $I(d)$ process, to model and forecast time series, where the differencing parameter $d$ is a fractional number between -0.5 and 0.5 . The main feature of the stationary $I(d)$ process is that its autocovariance function declines at a hyperbolic rate, slower than the geometric rate of stationary ARMA processes. For example, Ding et al. (1993) and Bollerslev and Mikkelsen (1996) showed that the persistence in stock market volatility could be well described by a long memory process. These findings further induced Hidalgo and Robinson (1996) to consider the issue of structural stability of a regression model with a long memory error term. However, Kuan and Hsu (1998) find that the Hildago and Robinson (1996) test could have large size distortions. Additionally, extending the results of Nunes et al. (1995), Kuan and Hsu (1998) show that the conventional break tests for stationary long memory processes may misleadingly infer a structural break when there is none. Because of the possibility of misleading inference by the existing structural change tests for long memory processes, scant attention has been paid to suggesting optimal methods to forecast long memory processes in the presence of structural breaks.

The purpose of this paper is to propose an easy-to implement approach for forecasting a long memory process that may be subject to structural breaks. The conventional forecasting method based on post-break data can be suboptimal because the break detection approach may lead to spurious conclusions concerning the number of breaks even there is none (e.g. Granger and Hyung, 2004). Moreover, Granger and Hyung (2004) and Choi et al. (2010) also showed that an increase of the number of mean breaks makes the memory of the process seemingly more persistent. Choi et al. (2010) use a break-adjusted forecast method to reduce the forecast error for several realized volatility series, which are modelled as long memory processes with breaks, however, their method still depends on the knowledge of the accurate break dates. To avoid the risk of misleading inference on the break date or imprecise estimation of the fractional parameter $d$, we note that first, Granger (1980) has shown that when a long memory process has a break in the parameter $d$, the complete time series can be represented by another long memory process with memory parameter $d^{*}$ that is a linear combination of the pre-and the post-break memory parameters $d_{1}$ and $d_{2}$. Second, Poskitt (2007) and Wang and Hsiao (2012) have shown that a stationary 
ARFIMA $(p, d, q)$ process can be approximated by an autoregressive process with an order increasing at a certain rate of the sample size $\mathrm{T}$. We therefore suggest using an autoregressive approximation to predict the outcome for long memory processes with breaks. We justify the use of an autoregression approximation when the time series follows an ARFIMA $(p, d, q)$ process, for two types of structural breaks: a change in the long memory parameter and/or a shift in mean when the shift size is within some magnitue of the standard deviation of the random noise. Furthermore, an AR approximation approach also has the advantage of avoiding the inaccurate parameter estimation and the break locations.

An important issue in finite sample is to select the appropriate order of the AR model. We suggest using Mallow's $C_{p}$ criterion to select the order of an $\operatorname{AR}(k)$ model fitted to a long memory process with structural change. Our Monte Carlo experiments show that the lag length based on Mallow's criterion to approximate the ARFIMA process with structural breaks is usually small in a sample of two hundred observations. and the residual variance estimate is very close to the true error variance. Our simulation experiments confirm the theoretical analysis clearly by demonstrating that an AR-approximation forecast method for forecasting a long memory process with structural breaks outperforms conventional methods, namely the two naive ARFIMA-based methods, the post-break and Tiao and Tsay (1994) adpative forecasting methods, even in cases where the structure of an ARFIMA model, including its parameters and lag orders, changes dramatically after breaks. Furthermore, for the special case in which the structural breaks take place immediately prior to the forecast period, our AR-approximation also performs better. An empirical forecasting exercise of realized volatility for the DEM/USD, USD/YEN and GBP/USD spot exchange rates shows that our AR-approximation dominates the existing methods.

We present the basic model and theoretical results in Section 2. Section 3 provides the mean-squared prediction errors of forecasts generated by an AR approximation, a post-break model, and two naive ARFIMA- based forecast models. Section 4 provides the finite sample simulation comparison. Section 5 provides the comparison of different methods for predicting volatilities. Concluding remarks are in Section 6 . Proofs are in the Appendix.

\section{The Model and Theoretical Results}

\subsection{The Basic Model}

Let

$$
\begin{aligned}
& (1-L)^{d_{1}}\left(\eta_{t}^{(1)}-\mu_{1}\right)=e_{t} \\
& (1-L)^{d_{2}}\left(\eta_{t}^{(2)}-\mu_{2}\right)=e_{t}
\end{aligned}
$$


be two $A R F I M A(0, d, 0)$ processes where (i) $d_{1}, d_{2} \in(-0.5,0.5), d_{1} \neq 0$ and $d_{2} \neq 0$ are differencing parameters; (ii) $e_{t}$ is an independently and identically distributed process, with $E\left(e_{t}\right)=0, E\left(e_{t}^{2}\right)=\sigma^{2}$, and $E\left(e_{t}^{4}\right)<\infty$. We suppose the $T$ time series observations, $\eta_{t}$ take the form

$$
\eta_{t}=\eta_{t}^{(1)} \quad \text { for } \quad t=1,2, \cdots, T_{1},
$$

and

$$
\eta_{t}=\eta_{t}^{(2)} \quad \text { for } \quad t=T_{1}+1, \cdots, T
$$

where $T_{1}=\kappa T, \kappa \in(0,1)$. We consider two scenarios:

Case I: Changes in the differencing parameter only, i.e., $d_{1} \neq d_{2}, \quad \mu_{1}=\mu_{2}=\mu$.

Case II: Changes in both differencing parameter and mean, i.e., $d_{1} \neq d_{2}, \quad \mu_{1} \neq \mu_{2}$.

We focus on breaks in the mean and long memory parameters in the DGP. We could, of course, examine ARFIMA $(p, d, q)$ models with breaks in the coefficients of the AR and MA terms. However, this would substantially complicate the notations and derivations without gaining insight.

Lemma 1. 1 When the DGP satisfies the basic model, with $T_{1}=\kappa T, \kappa \in(0,1)$, the observed data can be represented by a long memory process with long memory parameter $d^{*}$ that is a linear combination of the pre-and post-break parameters $d_{1}$ and $d_{2}$, and with a mean $\mu^{*}$ that is a linear combination of the pre-and post-break means $\mu_{1}$ and $\mu_{2}$, that is

$$
(1-L)^{d^{*}}\left(\eta_{t}-\mu^{*}\right)=e_{t}
$$

where $\mu^{*}=\kappa \mu_{1}+(1-\kappa) \mu_{2}, d^{*}=\lambda d_{1}+(1-\lambda) d_{2}, 0 \leq \lambda \leq 1$. When $T$ is finite, $\kappa \rightarrow 0, \lambda \rightarrow 0$, and $\kappa \rightarrow 1, \lambda \rightarrow 1$. When $T \rightarrow \infty, d^{*}=\max \left(d_{1}, d_{2}\right)$ and $\mu^{*}=\kappa \mu_{1}+(1-\kappa) \mu_{2}$.

Brockwell and Davis (1991) have shown that a long memory process can be represented by an infinite order autoregressive process,

$$
\eta_{t}=\mu+\sum_{j=1}^{\infty} \beta_{j} \eta_{t-j}+e_{t}
$$

where $\beta_{j}=\Gamma(j-d) /[\Gamma(j+1) \Gamma(d)], d \in(-0.5,0.5), d \neq 0$, and $e_{t}$ is a zero mean white noise process with constant variance $\sigma^{2}$. Poskitt (2007) and Wang and

1 Lemma 1 is a generalization of that of Granger (1980) with less restrictive conditions on the distribution of the DGP and also allows breaks in the mean. 
Hsiao (2012) have shown that $\eta_{t}$ can be approximated by an ever increasing order autoregressive model, $\mathrm{AR}(k)$, as $T$ increases,

$$
\eta_{t}=e_{t, k}+\sum_{j=1}^{k} \beta_{j k} \eta_{t-j}+\beta_{0 k}
$$

where $e_{t, k}$ is the prediction error and $\beta_{j k}, j=0,1,2, \cdots, k$ are the coefficients of the minimum mean squared predictor of $\eta_{t}$ based only on a constant term and the past observations $\eta_{t-1}, \eta_{t-2}, \cdots, \eta_{t-k}$.

THEOREM 1. ${ }^{2}$ When the DGP satisfies the basic model, with $T_{1}=\kappa T, \kappa \in$ $(0,1)$, when $d^{*} \in(-0.5,0.5)$ there exists an $A R(k)$ approximation of $\eta_{t}$, as $T \rightarrow \infty$, $k=O\left(T^{r}\right), r>2 d^{*} /\left(1+2 d^{*}\right)$, such that

1. $\|\widehat{\beta}(k)-\beta(k)\|=O_{p}\left(k^{-1 / 2}(\log T)^{d^{*}-0.5} T^{-d^{*}}\right)$,

2. $\widehat{\sigma}_{t, k}^{2}=\frac{1}{T-k} \sum_{t=k+1}^{T} \widehat{e}_{t, k}^{2}=\sigma^{2}+O_{p}\left(k^{-2 d^{*}-1} T^{2 d^{*}}\right)=\sigma^{2}+o_{p}(1)$, where $\widehat{\beta}(k)$ is the OLS estimator of $\beta(k)$ and $\widehat{e}_{t, k}$ the OLS residual.

Given Lemma 1 and Theorem 1, we suggest using an $\operatorname{AR}(k)$ model to approximate the data generating process of $\eta_{t}$ and using it to generate post-sample predictions.

\subsection{Mallows' $C_{p}$ Criterion for Choosing $k$}

Theorem 1 says that $k \rightarrow \infty$ as $T \rightarrow \infty$. As shown in Shibata (1980), for each $T$, there is an optimal $k^{*}$ to balance the magnitude of the estimation error and bias of the finite $\operatorname{AR}(k)$ approximation. In other words, the adequacy of an approximate model for the DGP depends on the choice of the lag order. Therefore, in this paper we propose using AIC (Akaike, 1973) or $C_{p}$ (Mallows, 1973) criterion to choose a suitable order $k^{A I C}$ or $k^{C_{p}}$ of an $\mathrm{AR}(k)$ model to approximate a long memory process subject to a break of the kind defined for our basic model.

Mallows' criterion is based on choosing the lag length to minimize the expected mean-squared prediction error divided by the variance of the DGP. Because the complete time series can be represented by another long memory process with parameter $d^{*}$, when a long memory process has a break in the long memory parameter, by Poskitt (2007) and Wang and Hsiao (2012), we note the AIC and $C_{p}$ criteria are simply

$$
A I C(k)=\log \widehat{s}_{t, k}^{2}+\frac{2 k}{T}
$$

2 The rate of convergence of $\|\widehat{\beta}(k)-\beta(k)\|$ is shown faster than that of Poskitt (2007) or Wang and Hsiao (2012) because we use a different methodology . 
and

$$
C_{p}(k)=(T-k)\left(\frac{\widehat{\sigma}_{t, k}^{2}}{\widehat{\sigma}^{2}}-1\right)+2 k,
$$

where $\widehat{\sigma}^{2}$ is a consistent estimator of $\sigma^{2}$ (or the true value in a simulation setup).

It appears that a suitable procedure for choosing an appropriate $k^{*}$ for an $\operatorname{AR}(k)$ approximation to our basic model is to minimize $A I C(k)$ or $C_{p}(k)$ with respect to $k$. More specifically, following the analysis of Shibata (1980), Poskitt (2007) showed that $A R\left(k^{A I C}\right)$ and $A R\left(k^{C_{p}}\right)$ model are asymptotically efficient. We conducted Monte Carlo experiments about the choice of $k$ using these two criteria for several DGPs and sample sizes. Results are nearly identical between AIC and $C_{p}$, as in Wang and Hsiao (2012) and are availble upon request. The main conclusion is that when $T=200$, a value of $k^{C_{p}}$ not larger than 4 often provides a good AR-approximation of long memory processes subject to one or two breaks, which are representative of empirical models reported in the literature.

\section{Comparison of Forecasting Methods}

The usual approaches to forecasting a long memory process with structural breaks are the "post-break (PB)" and "naive" ARFIMA-based forecast (NV) methods. The post-break method uses an estimated ARFIMA model after the last identified break date. The " naive " ARFIMA-based (NV) forecast method first tests for structural breaks. If there is a break in the mean or the long memory parameter, but no break in the AR or MA operators, both pre-and post-break mean filtered data are used to estimate the posk-break ARFIMA model (Hyung and Franses, 2001) ${ }^{3}$. The resulting model is then used to generate forecasts. In other words, if there are breaks both in the mean and the long memory paramter $d$, the pre-and post-break means and long memory parameters are used to generate forecasts. If no break is detected, forecasts are computed using the estimated ARFIMA model using the complete data. Furthermore, inspired by Lemma 1, we can ignore the pretest step and simply use an alternative naive $\operatorname{ARFIMA}\left(d^{*}\right)$ model to generate forecasts (we call this method NVNO, for "nave-no break"). More specifically, the NVNO method ignores any detected break and uses the complete data to estimate the ARFIMA model generate forecasts. The NVNO method thus coincides with the NV method only when no break is detected.

In this section, we show by a theoretical argument that $h$-step ahead forecasts of a long memory process subject to a structural break computed on the basis of our AR-approximation method dominate, in the mean-squared error sense, those of

3 They only consider the case of a mean shift. 
the post-break and naive ARFIMA-based forecast methods. Our analysis is based on selecting the minimum mean-squared error of the point forecast of $\eta_{t+h}$ in the context of our basic model.

We assume the post-sample $\eta_{t}$ is generated from $(1-L)^{d_{2}}\left(\eta_{t}^{(2)}-\mu_{2}\right)=e_{t}$. When there is no structure break for the observed $\eta_{t}, t=1,2,3, \cdots, T, \kappa=0, \mu^{*}=\mu_{2}$ and $d^{*}=d_{2}$. In deriving the mean square prediction error of different forecasting methods, we assume $T_{1}$ is known and the post-break window size, $T-T_{1}=(1-\kappa) T=$ $T_{2}$, is not too large, because otherwise the forecast of $\eta_{T+h}$ will just be dominated by post-break observations without loss of important information.

Following Lemma 1, $\eta_{t}$ can be written as

$$
\eta_{t}=(1-L)^{d^{*}} \mu^{*}+\sum_{j=1}^{\infty} \beta_{j} \eta_{t-j}+e_{t}, \quad \text { where } \quad \beta_{j} \sim C j^{-d^{*}-1}
$$

Approximating (6) by an $\mathrm{AR}(k)$ model yields

$$
\left(1-\sum_{j=1}^{k} \beta_{j k} L^{j}\right) \eta_{t}=\left(1-\sum_{j=1}^{k} \beta_{j k} L^{j}\right) \mu^{*}+e_{t}=\beta_{0 k}+e_{t},
$$

where $\beta_{0 k}=\left(1-\sum_{j=1}^{k} \beta_{j k}\right) \mu^{*}$ and $E\left(\eta_{t}\right)=\mu^{*}$. Hence, the $h$-step ahead forecast based on the $\operatorname{AR}(k)$ approximating model is

$$
\widehat{\eta}_{T+h}=\widehat{\beta}_{0 k}+\sum_{j=1}^{k} \widehat{\beta}_{j k} \eta_{T+h-j} .
$$

The $h$-step ahead predictor $\widetilde{\eta}_{T+h}$ of the naive ARFIMA-based method can be computed recursively by using $\widetilde{\pi}_{1}(L)=\sum_{j=0}^{\infty} \widetilde{\pi}_{1, j} L^{j}=(1-L)^{\widetilde{d}_{1}}, \pi_{1, j}=\frac{\Gamma\left(j-\widetilde{d}_{1}\right)}{\Gamma(j+1) \Gamma\left(-\widetilde{d}_{1}\right)}$ and $\widetilde{\pi}_{2}(L)=\sum_{j=0}^{\infty} \widetilde{\pi}_{2, j} L^{j}=(1-L)^{\widetilde{d}_{2}}, \pi_{2, j}=\frac{\Gamma\left(j-\widetilde{d}_{2}\right)}{\Gamma(j+1) \Gamma\left(-\widetilde{d}_{2}\right)}$, where $\widetilde{\pi}_{1, j} \sim j^{-\widetilde{d}_{1}-1} / \Gamma\left(-\widetilde{d}_{1}\right)$ and $\widetilde{\pi}_{2, j} \sim j^{-\widetilde{d}_{2}-1} / \Gamma\left(-\widetilde{d}_{2}\right)$ denote the forecast coefficients based on the estimated memory parameter $\widetilde{d}_{1}$ and $\widetilde{d}_{2}$ before and after the break, respectively. In other words, we need to estimate $d_{1}$ and $d_{2}$ first and then plug them into the formula of $\pi_{1, j}$ and $\pi_{2, j}$. For the conventional ARFIMA-based prediction approach, there could be two possibilities-a break is detected or not detected. Suppose there is a break at $T_{1}=\kappa T, \kappa \in(0,1)$ for the observed $\eta_{t}$. If we do not detect an existing break by a structural break test, we use a naive ARFIMA-based model, which is similar to the NVNO method, ${ }^{4}$ with a long memory parameter $d^{*}$ and $\mu^{*}$ to generate the forecast.

4 For ease of exposition, we follow Brockwell and Davis (1991) to generate forecast based on all observations $(\mathrm{T})$. In other words, we do not consider a truncated order $\mathrm{k}$ fitted to the observed time series. 
Explicitly,

$$
\widetilde{\eta}_{T+h}=(1-L) \widetilde{d}^{*} \widetilde{\mu}^{*}+\sum_{j=1}^{T} \widetilde{\pi}_{j}^{*} \eta_{T+h-j}
$$

where $\widetilde{\pi}_{j}^{*} \sim j^{-\widetilde{d}^{*}}$ is derived from the estimated parameter $\widetilde{d}^{*}$.

On the other hand, if a break is detected at $T_{1}$, then $\eta_{t}=\eta_{t}^{(2)}$ for data generated by the post-break model $e_{t}=(1-L)^{d_{2}}\left(\eta_{t}^{(2)}-\mu_{2}\right), \quad$ for $\quad t \geq T_{1}+1$. Specifically,

$$
\eta_{T+h}=(1-L)^{d_{2}} \mu_{2}+\sum_{j=1}^{T_{2}} \pi_{2, j} \eta_{T+h-j}^{(2)}+\sum_{j=T_{2}+1}^{T} \pi_{2, j} \eta_{T+h-j}^{(2)}+e_{T+h}
$$

However, $\eta_{t}^{(2)}$ for $t$ before $T_{1}+1$ are unobservable. On the other hand, we do know $e_{t}$ for $t \leq T_{1}$, which is equal to $(1-L)^{d_{1}}\left(\eta_{t}^{(1)}-\mu_{1}\right)=e_{t}$, for $t=1,2, \cdots, T_{1}$. Since $\eta_{t}^{(2)}=\mu_{2}+(1-L)^{-d_{2}} e_{t}$ and $d_{1}-d_{2}=d_{3}$, therefore, for $t$ before $T_{1}+1$,

$$
\eta_{t}^{(2)}=\mu_{2}+(1-L)^{-d_{2}}(1-L)^{d_{1}}\left(\eta_{T+h-j}^{(1)}-\mu_{1}\right)=\mu_{2}+(1-L)^{d_{3}}\left(\eta_{T-j+h}^{(1)}-\mu_{1}\right) .
$$

Thus, $\eta_{T+h}$ expressed in terms of $\eta_{t}, t=1,2, \cdots, T$, becomes

$$
\begin{aligned}
& \eta_{T+h}=(1-L)^{d_{2}} \mu_{2}+\sum_{j=1}^{T_{2}} \pi_{2, j} \eta_{T+h-j} \\
& +\sum_{j=T_{2}+1}^{T} \pi_{2, j}\left((1-L)^{d_{3}}\left(\eta_{T+h-j}-\mu_{1}\right)+\mu_{2}\right)+e_{T+h} .
\end{aligned}
$$

Let $a$ and $1-a$ be the probability of finding no break (type II error) and a break, respectively. Let $\pi_{j}^{*}, \pi_{1, j}, \pi_{2, j}$ denote the forecast coefficients derived from estimated $d^{*}, d_{1}$ and $d_{2}$, i.e., $\widetilde{d}^{*}, \widetilde{d}_{1}, \widetilde{d}_{2}$. Then the predictor for $\eta_{T+h}$ based on the naive forecast approach becomes

$$
\begin{aligned}
& \widetilde{\eta}_{T+h}^{I I}=a\left((1-L)^{\widetilde{d}^{*}} \widetilde{\mu}^{*}-\sum_{j=1}^{T} \widetilde{\pi}_{j}^{*} \eta_{T+h-j}\right)+(1-a)\left((1-L)^{\widetilde{d}_{2}} \widetilde{\mu}_{2}+\sum_{j=1}^{T_{2}} \widetilde{\pi}_{2, j} \eta_{T+h-j}\right. \\
& \left.+\sum_{j=T_{2}+1}^{T}(1-L)^{\widetilde{d}_{3}} \widetilde{\pi}_{2, j} \eta_{T+h-j}-\sum_{j=T_{2}+1}^{T}(1-L)^{\widetilde{d}_{3}} \widetilde{\pi}_{2, j} \mu_{1}+\sum_{j=T_{2}+1}^{T} \widetilde{\pi}_{2, j} \mu_{2}\right) .
\end{aligned}
$$

In the event there is no break in the observed $\eta_{t}$, i.e., $\kappa=0, \eta_{t}=\eta_{t}^{(2)}$, let $b$ denote the probability of finding no break and $1-b$ denote the probability of finding 
a break (type I error). Then the naive forecast for $\eta_{T+h}$ is equal to

$$
\begin{aligned}
& \widetilde{\eta}_{T+h}^{I}=b\left((1-L)^{\widetilde{d}_{2}^{*}} \widetilde{\mu}_{2}^{*}+\sum_{j=1}^{T} \widetilde{\pi}_{2, j}^{*} \eta_{T+H-j}\right)+(1-b)\left((1-L)^{\widetilde{d}_{2}} \widetilde{\mu}_{2}+\sum_{j=1}^{T_{2}} \widetilde{\pi}_{2, j} \eta_{T+h-j}\right. \\
& \left.+\sum_{j=T_{2}+1}^{T}(1-L)^{\widetilde{d}_{3}} \widetilde{\pi}_{2, j} \eta_{T+h-j}-\sum_{j=T_{2}+1}^{T}(1-L)^{\widetilde{d}_{3}} \widetilde{\pi}_{2, j} \mu_{1}+\sum_{j=T_{2}+1}^{T} \widetilde{\pi}_{2, j} \mu_{2}\right),
\end{aligned}
$$

where $\left(\widetilde{d}_{2}^{*}, \widetilde{\mu}_{2}^{*}\right)$ and $\left(\widetilde{d}_{2}, \widetilde{\mu}_{2}\right)$ are based on MLE estimates of $\mu_{2}$ and $d_{2}$ based on $\mathrm{T}$ and $T_{2}=(1-k) T$ observations, respectively.

We also consider the case that the exact break point is known and use only post break data to estimate the post-break ARFIMA model and generate forecasts (PB). The $h$ - step ahead forecast of post-break method is just

$$
\widehat{\eta}_{T+h}^{*}=\left((1-L)^{\widehat{d}_{2}} \widehat{\mu}_{2}+\sum_{j=1}^{T_{2}} \widehat{\pi}_{2, j} \eta_{T+h-j}\right) .
$$

The following theorem shows the comparison of the $h$ - step ahead mean squared error for four forecasting methodologies.

THEOREM 2. If the DGP satisfies the basic model, (i) the AR(k) approximationbased forecast method (AR) yields no greater mean squared error than the postbreak forecast and the two naive ARFIMA-based forecast ( $N V$ and NVNO) method if $\kappa\left|\mu_{1}-\mu_{2}\right|$ is of smaller order than $\sigma_{\eta} / \sqrt{(1-\kappa) T}$, where $\sigma_{\eta}$ denotes the standard error of $\eta_{t}$; (ii) when $k=O\left((\ln T)^{-1}(T / \ln T)^{1-2 d^{*}}\right)$, the AR outperforms ARFIMA(d*) (NVNO).

Tiao and Tsay (1994) and Baillie and Morana (2009) suggest an adaptive forecasting approach to account for both long memory and structural change in the conditional variance process. The basic idea of their approach is to obtain the estimates by minimizing the sum of squares of $h$-step-ahead forecast errors. More specifically, as each additional post-sample time series observation becomes available, the parameters of the forecasting model is recursively re-estimated by minimizing the sum of squares of $h$-step-ahead forecast errors based on the rolling window with a fixed window size where with each additional avaiable observation, the observation at the beginning of the time period is dropped. This approach would be inefficient ${ }^{5}$ compared to recursively updating the parameter estimates when each additional observation becomes available if there is no structural break. On the other hand, if

5 Tiao and Tsay (1994) points out the adpative forecasting would be inefficient if the model used is indeed the " correct" one. 
there is indeed a structural break at time period $T_{1}$, as more and more post-sample information becomes available, this is equivalent to pushing the break point $k$ towards zero in Lemma 1 and Theorem 2. In other words, our AR approximaiton-based forecast aprroach under Tiao and Tsay (1994) and Baillie and Morana (2009)'s adaptive forecasting scheme, denoted as AFAR, yields

Lemma 2. If the DGP satisfies the basic model (with a fixed break data $T_{1}$ ), the adaptive AR approximation-based forecast method (AFAR) with a rolling window (with fixed window size) eventually yields no greater mean squared error than the post-break forecast and the two naive ARFIMA-based forecast (NV and NVNO) methods for Case I and for Case II.

Theorem 2 and Lemma 2 illustrate the performance of the two AR approximationbased forecast method (AR and AFAR) that are limited to the case where the DGP has a single structural break. Although the analysis can be extended to the cases of more breaks, the extension is tedious to show. Additionally, Poskitt (2007) points out that estimating the parameters of the AR approximation model by directly minimizing the observed mean squared error could lead unstable estimates. Thus, AR and AFAR could exhibit quite different finite sample behaviour. We therefore resort to Monte Carlo experiments to provide intuition on these extensions, and also to illustrate the difference in performance of the different forecasting methods for the $h$-step forecasts under a single break.

\section{Comparison of Forecasting Methods: Simulation Results}

\subsection{Simulation Design}

In light of the empirical evidence provided by Christensen and Nielsen (2006), and Choi et al. (2010), we consider

$$
\begin{array}{ll}
\operatorname{DGP}(\mathrm{a}): & (1-L)^{d}\left(\eta_{t}-\mu\right)=e_{t}, \\
\operatorname{DGP}(\mathrm{b}): & (1-0.7 L)(1-L)^{d}\left(\eta_{t}-\mu\right)=(1+0.5 L) e_{t}, \\
\operatorname{DGP}(\mathrm{c}): & \left(1-0.7 L-0.2 L^{2}\right)(1-L)^{d}\left(\eta_{t}-\mu\right)=e_{t} .
\end{array}
$$

We let the above DGPs be subject to breaks in the mean and in the long memory parameter, each being considered with $d=(0.1,0.2,0.3,0.4,0.45)$ and $\sigma^{2}=1$, see Tables 1 and 2 for the values of the break point, means and differencing parameters. Simulations are performed for $T=200$ and repeated 1000 times. Following McLeod and Hipel (1978) and Hosking (1984), we first generate $T$ independent $\mathrm{N}(0,1)$ values and form a $T \times 1$ vector $e$, then generate the vector $y$ of the $T$ realized values of the $I(d)$ process as $y=C e$, where $\mathrm{C}$ is the Cholesky decomposition of the $T \times T$ 
autocovariance matrix $\Sigma=C C^{\prime}$. For each $T$, we generate $T+200$ observations and discard the first 200 to reduce the effects of initial values.

For each experiment, we assume the break dates are either known or unknown. In the case of unknown break points, tests proposed by Bai and Perron (1998) and Beran and Terrin (1996) are used to detect the change points. We then use the Schwarz information criterion to select the number of breaks for each model. To estimate the differencing parameter $d$, we use the GPH and the approximate ML estimation methods. Both methods provide similar results confirming that that the higher $p$ in the ARFIMA $(p, d, q)$ DGP, the larger the bias of the estimate of $d$.

We consider both AR forecasting and the AFAR forecasting procedure. The AFAR is the combination of the adaptive forecasting scheme and our AR approximation. If the AR model we employ here is indeed the " correct " one (the estimate of $\sigma^{2}$ converges to $\sigma^{2}$ asympototically), then it would be inefficient to use the adaptive forecasting (see Tiao and Tsay, 1994). Additionally, we also compute forecasts using the naive $\operatorname{ARFIMA}\left(d^{*}\right)$ model (3) without concern for the break detection (NVNO). We compare the out-of-sample forecasting performance of the three forecast methods described in Section 3 and that of the AFAR and NVNO methods for two forecasting horizons: $h=1,5$.

The recursive forecast window scheme is designed as follows: given a simulated sample of size $T=2 \widetilde{T}$, we estimate the model using the first half of the sample, and generate the forecasts of $\eta_{\widetilde{T}+h}$ for $h=1,5$. Then we add one observation to the estimation sample, and generate the forecasts of $\eta_{\widetilde{T}+1+h}$. We continue like this until observation $T-h$, which enables to forecast $\eta_{T}$. We denote by $\eta_{\widetilde{T}+f+h}^{M}$ the $h$-step ahead forecast of $\eta_{\widetilde{T}+f+h}$ using model $M$ and information up to date $\widetilde{T}+f$. The relative RMSFE of model $M$ with respect to the $\operatorname{AR}(k)$ model, for $\tilde{T}$ forecasts at horizon $h$, is defined as

$$
\frac{\left(\sum_{f=0}^{\widetilde{T}-h}\left(\eta_{\widetilde{T}+f+h}-\eta_{\widetilde{T}+f+h}^{A M}\right)^{2}\right)^{1 / 2}}{\left(\sum_{f=0}^{\widetilde{T}-h}\left(\eta_{\widetilde{T}+f+h}-\eta_{\widetilde{T}+f+h}^{A R}\right)^{2}\right)^{1 / 2}}
$$

The value of $k$ for the $\mathrm{AR}(k)$ model is chosen by Mallows' criterion. Table 3 reports the root mean squared forecast errors (RMSFE) of the naive, naive-no and postbreak methods, relative to that of the AR-based method with recursive forecasting window. We further consider the rolling estimation window. The window size is based on the suggestion of Hansen and Timmermann (2012) to use half of the full sample for in-sample estimation (=100 observations), and the remaining for out-of-sample 
evaluation. ${ }^{6}$ Those relevant results are reported in Table 4. If the relative RMSFE is smaller than one, the alternative model forecasts better than the AR model.

To further determine which model provides more accurate information about the future, we use the Mincer and Zarnowitz (1969) encompassing regression for the one-step-ahead forecasts of four forecast models with the rolling forecast window:

$$
\eta_{t+1}=\alpha_{0}+\alpha_{1} \eta_{t+1 \mid t}^{A R}+\alpha_{2} \eta_{t+1 \mid t}^{A M}+\varepsilon_{t}
$$

where $\eta_{t+1 \mid t}^{A R}$ denotes predictons by our benchmark AR approximation model, and $\eta_{t+1 \mid t}^{A M}$ denotes predictions from four alternative models. The results in Tables 3 and 4 show that the forecasts of the AR-approximation generally outperform the alternative methods. We find that :

(1) Unknown or known break dates: the AR approximation ignoring the possibility of breaks almost always generates more accurate one-step ahead forecasts. For the 5 -period ahead forecasts, there are very few cases where post-break forecasts are more accurate with unknown (PBUK) than with known break dates (PBK). However, if break dates are unknown, which is the most relevant situation in empirical studies, the AR-based forecast method dominates other methods in 3 out of 15 experiments for all considered forecast horizons and forecast windows. The domination is substantial, with frequently occurring gains in RMSFE of more than 20 per cent. More precisely, although using the rolling window sometimes reduces the RMSFE of the compared models for several cases (experiment 1,2,6,7,8 and 12), the AR-based forecast method in general still performs the best.

(2) (i) AR compared to NVNO: for most cases, AR dominates uniformly, except in experiment 11 for horizon $h=5$. More interestingly, for the cases where the series properties shift (experiment 1, 2, 7,8 and 15), the NVNO even perfoms the worst among all forecast methods, although the rolling window could reduce its RMSFE. These results could be due to the inaccurate estimation of the NVNO model in finite samples. (ii) AR compared to the AFAR method: for most cases, AR performs much better than ARFA method, except for the experiments $3,4,5,9,10$ and 13 , where we only consider a change in the long memory parameters and rolling window estimation. However, the AFAR performs better than the other five methodologies. We further caculate the average (AVE) of RMSFE for each $h$ and forecast method with two different forecast windows. The results do not change.

(3) The results in Table 5 show the superior forecasting ability of the AR model. In the encompassing regression that includes both the AR approximation-based forecast model and an alternative forecast, the estimates of $\alpha_{1}$ are close to unity

6 We thank the editors for this suggestion. 
and the estimates for $\alpha_{2}$ are close to zero whether the break date is known or unknown. We also use a $F$-test for the null hypothesis of $\alpha_{0}=0, \alpha_{1}=1$ and $\alpha_{2}=0$ at the $5 \%$ significant level. The P-values of the $F$-tests $(\mathrm{P}(F))$ are all above $15 \%$. Furthermore, including an alternative forecast method has little contribution to increasing $R^{2}{ }^{7}$

\subsection{Comparison When the Break Occurs at the End of the Sample}

Forecasting observations of long memory processes just after a structural break is a difficult task. The post-break forecast method cannot be implemented in this case, due to the absence of informative observations about the post-break period. The naive ARFIMA-based method can be used with estimates from the pre-break period, being in addition prone to the spurious break and inaccurate parameter estimation problems. Moreover, in empirical research, it is difficult to know exactly when structural breaks occur. Thus, the existing forecasting approaches are ill-suited for real-time forecasting. Given that a structural break occurs at $T_{1}$, we present in Table 7 the performance of the AR-approximation (AR), AFAR and NV of observations $T_{1}+h$ for $h=1,2, \ldots, 5$, assuming we do not know the existence of the break to generate the forecasts. The results clearly indicate that the AR-approximation method produces the lowest RMSFEs.

In closing this section, it is worth pointing out that the superior performance of our AR-approximation forecast method goes along with its ease of implementation in empirical analysis. Intuitively, the reason why the AR-based forecasting method produces the best performance is that it avoids the inaccurate estimation of the fractional parameters and the issue of spurious breaks induced by long memory processes. 8

\section{Forecasting Realized Volatilities}

Volatility forecasting plays a central role in financial decision making, for example, fund managers adjust the portfolio allocations according to the volatility forecasts. Choi et al. (2010) use a break-adjusted forecast method to reduce the forecast error

\footnotetext{
7 For the encompassing regression, the first forecast is said to subsume information in other forecasts if these additional forecasts do not significantly increase the $R^{2}$.

8 The resson why the forecasting performance of $\operatorname{ARFIMA}\left(d^{*}\right)(\mathrm{NVNO})$ is worse than that of AR in finite samples may be the difficulty of accurately estimating the parameter $d$ when it is close to 0.5 and the sample size is not large ( $\mathrm{T}=100$ and 200). Furthermore, it is also hard to distinguish the behaviour of long and short range components for data with both components in finite samples (See Crator and Ray, 1996).
} 
for several realized volatility series, which are modelled as long memory processes with breaks. In order to show the potential usefulness of our AR-approximation based forecasting method, we compare the forecasts of our method to the four competing methods considered in Section 3, the heterogeneous autoregressive (HAR) model of Corsi (2004), and the VAR-RV-Break model proposed by Choi et al. (2010). The HAR model is actually an AR model with only three coefficients for 21 lags, plus a constant term, which can be written

$$
R V_{t}=\alpha_{0}+\alpha_{1} R V_{t-1}+\alpha_{2} \sum_{i=1}^{5} \frac{1}{5} R V_{t-i}+\alpha_{3} \sum_{j=1}^{21} \frac{1}{21} R V_{t-j}+u_{t}
$$

Corsi's justification for this model was to capture the long memory property which he justifies by an economic argument. Many researchers have found empirically this model to fit and to forecast realized volatility series better than ARFIMA models. The HAR model can be seen as a particular case of our AR approximation, where the lag order is fixed to 21 whatever the sample size, and the coefficients are constrained. Our theoretical and simulation results in this paper explain to a large extent, from an econometric viewpoint, why the HAR model is a successful model in the context of realized volatility modelling.

The VAR-RV-Break (VRB) model is a break-adjusted VAR, denoted as

$$
\Phi(L)\left(Y_{t}^{*}-\mu\right)=\varepsilon_{t},
$$

where $Y^{*}$ is the $(3 \times 1)$ vector of logarithmic realized exchange rate volatilities after mean break adjustments, $\mu$ is the unconditional mean, $\varepsilon_{t}$ is a vector white noise process and the lag order is equal to five following Choi et al. (2010). The detailed procedure of the mean break adjustments is explained in Choi et al. (2010).

We perform the comparisons for the realized volatility series of the spot exchange rate returns of the German mark (DEM) and British pound (GBP) versus the US dollar, and the US dollar versus the Japanese Yen (YEN). The data (obtained from Olsen \& Associates) span the period starting on January 6, 1987 and ending on June 30, 1999. They consist of fifteen-minute returns, which we square and aggregate to construct the daily realized volatility. We prepared the data in a way similar to Andersen et al. (2001), who use the same series but their sample ends on November 30, 1996, and Andersen et al. (2003) and Choi et al. (2010) who use the intra-day 30-minute log returns. We transform the realized volatility series in logarithm since this ensures the positivity of forecasts of realized volatility. For simplicity, we denote by $R V_{t}$ the log of realized volatility for day $t$. The summary statistics are in Table 7. Unconditionally, the series have a positive skewness and a little too much excess kurtosis to be characterized as Gaussian.

To detect structural break dates, we use the procedure of Beran and Terrin (1996). Table 8 reports the estimated break dates. Four beaks are found for GBP and five 
for the other series. Thus, for example, there are six regimes for the DEM series, the first one spanning the period from January 6, 1987 to May 22, 1989. The break dates are almost identical for DEM and GBP except that the break of November 3, 1993 for the DEM is not found for the GBP. The Yen shares two breaks with the other series (May 22, 1989 and June 30/July 1, 1997).

To implement the post-break and out-of-sample NV and NVNO forecast methods, we need an $\operatorname{ARFIMA}(p, d, q)$ model for each series. We estimated several models using the full sample and each regime by Beran's approximate ML method, and we selected the best model according to Akaike's information criterion. The results are available on request. The lag orders $p$ and $q$ of the selected models are all either 0 or 1 , and the estimated fractional differencing parameters suggest that the series are stationary but have indeed a long memory component (the estimates are between 0.29 and 0.49).

Forecasts are obtained using a rolling estimation window. We follow the suggestion of Hansen and Timmermann (2012) to use half of the full sample for in-sample estimation, and the remaining for out-of-sample evaluation. For the AR-approximation based method, the naive ARFIMA with break method, the naive ARFIMA without break method and the VAR-RV-Break model, the in-sample period goes from January 6, 1987 to Jan 10, 1992 for DEM (1528 observations), Jan 13, 1992 for GBP (1532 observations), and Jan 16, 1992 (1536 observations) for YEN. We estimate the models over these samples and generate forecasts at horizon $h=1$ and 3. Then we delete the first observation and add the next observation to the estimation sample to generate again the two forecasts. The model is re-estimated and the procedure is repeated until the last observation of June 1999.

The lag orders for the approximating $\operatorname{AR}(k)$ models of the three series, selected by Mallows $C_{p}$ criterion, are equal to 18 for for DEM, 19 for GBP, and 18 for Yen RV series. As these values remain the same for the full samples and for the in-sample periods, we kept them fixed throughout the prediction period. These values are close to the 21 lags suggested by Corsi. They are much larger than in our simulation experiments, because the sample size we are dealing with in this application is much higher (about 1522 versus 200).

To compare the predictive ability of the five methods at forecast horizons one and three, we use the RMSFE of the five methods, relative to the RMSFE of the ARapproximation based method. The definition is the same as in equation (11). Since all relative RMSFE are above 1, the results, reported in Table 9, indicate that our AR-approximation based forecast method outperforms the other methods. This is especially spectacular for the post-break method, the naive methods (NV and NVNO) and the VAR-RV-Break model (VRB). Corsi's HAR model involves a small efficiency loss (between 3 and 7 per cent).

We examine the explanatory power of the one period ahead forecasts for the six forecasting models by the Mincer and Zarnowitz encompassing regression. Table 10 shows that in the encompassing regression, the estimates of $\alpha_{1}$ are close to unity and the estimates of $\alpha_{2}$ are close to zero. Furthermore, these additional forecasts do not significantly increase the $R^{2}$. Under the null hypothesis that $\alpha_{0}=0, \alpha_{1}=1, \alpha_{2}=0$, the $\mathrm{P}$-values of the $F$-tests are all above $15 \%$. Hence these results confirm the better performance of the forecasts based on the AR-approximation. 


\section{Conclusions}

Forecasting is often a difficult task, especially in a rapidly changing world where structural changes occur. Given that some macroeconomic and financial time series display high persistence and signs of structural breaks, it is important to develop adequate forecasting methods. However, there could be differences between the structural analysis and the forecast. In the structural analysis, the aim is to identify the data generating process and obtain the efficient inference on unknown parameters. In forecasting, because of unknown breaks and the size of breaks, the focus should be on the robustness of forecasting procedures. In this paper, we have developed a simple to implement, and improved forecasting method based on an AR-approximation to forecast a long memory process subject to structural breaks. Additionally, we have proved theoretically and illustrated by Monte Carlo simulations that i) a stationary long memory $\operatorname{ARFIMA}(p, d, q)$ process subject to structural breaks can be well approximated by an $\mathrm{AR}(k)$ model when $k$ is chosen appropriately, and ii) our AR-based forecast method provides a better out-of-sample forecast performance than conventional methods for forecasting long memory processes under structural breaks. The root of the success of this new method is that it avoids the inaccurate estimation of parameters inherent to the other methods, and the problem of spurious breaks. The method is shown to be useful for forecasting the daily realized volatilities of exchange rate series.

Table 1. Breakpoint Specifications by Experiment (EX) (One Break)

\begin{tabular}{cccccccc}
\hline \hline EX & $D G P(I)$ & $D G P(I I)$ & $\tau$ & $d_{1}$ & $d_{2}$ & $\mu_{1}$ & $\mu_{2}$ \\
\hline 1 & $S D$ & $(a)$ & 0.5 & 0.0 & 0.1 & 0.0 & 0.0 \\
2 & $S D$ & $(b)$ & 0.5 & 0.0 & 0.1 & 0.0 & 0.0 \\
3 & $(a)$ & $(a)$ & 0.25 & 0.3 & 0.45 & 0.0 & 0.0 \\
4 & $(a)$ & $(a)$ & 0.5 & 0.3 & 0.45 & 0.0 & 0.0 \\
5 & $(a)$ & $(a)$ & 0.75 & 0.3 & 0.45 & 0.0 & 0.0 \\
6 & $(a)$ & $(a)$ & 0.5 & 0.3 & 0.45 & 0.3 & 0.9 \\
7 & $(a)$ & $(b)$ & 0.5 & 0.1 & 0.4 & 0.0 & 0.0 \\
8 & $(a)$ & $(b)$ & 0.5 & 0.1 & 0.4 & 0.3 & 0.9 \\
9 & $(b)$ & $(b)$ & 0.25 & 0.3 & 0.45 & 0.0 & 0.0 \\
10 & $(b)$ & $(b)$ & 0.75 & 0.3 & 0.45 & 0.0 & 0.0 \\
11 & $(b)$ & $(b)$ & 0.5 & 0.3 & 0.45 & 0.3 & 0.9 \\
12 & $(b)$ & & & & & & \\
& & & & & & & \\
\hline
\end{tabular}

Notes: $S D \sim N(0,1)$. 
Table 2. Breakpoint Specifications by Experiment (EX) (Two Breaks)

\begin{tabular}{cccccccccccc}
\hline \hline EX & $D G P(I)$ & $D G P(I I) D G P(I I I)$ & $\tau_{1}$ & $\tau_{2}$ & $d_{1}$ & $d_{2}$ & $d_{3}$ & $\mu_{1}$ & $\mu_{2}$ & $\mu_{3}$ \\
\hline 13 & $(a)$ & $(a)$ & $(a)$ & 0.3 & 0.6 & 0.0 & 0.4 & 0.2 & 0.0 & 0.0 & 0.0 \\
14 & $(a)$ & $(a)$ & $(a)$ & 0.3 & 0.6 & 0.1 & 0.3 & 0.45 & 0.3 & 0.9 & 0.75 \\
15 & $(a)$ & $(b)$ & $(c)$ & 0.3 & 0.6 & 0.1 & 0.3 & 0.45 & 0.3 & 0.9 & 0.75 \\
\hline \hline
\end{tabular}


Table 3. Relative Root Mean Squared Forecast Error (Recursive Window)

\begin{tabular}{|c|c|c|c|c|c|c|c|}
\hline$E X$ & $h$ & $P B K$ & $N V K$ & $P B U K$ & NVUK & $N V N O$ & $A F A R$ \\
\hline 1 & $\frac{1}{5}$ & $\begin{array}{l}1.172 \\
1.311\end{array}$ & $\begin{array}{l}1.383 \\
1.373\end{array}$ & $\begin{array}{l}1.258 \\
1.362\end{array}$ & $\begin{array}{l}1.422 \\
1.438\end{array}$ & $\begin{array}{l}1.479 \\
1.522\end{array}$ & $\begin{array}{l}1.138 \\
1.145\end{array}$ \\
\hline 2 & $\frac{1}{5}$ & $\begin{array}{l}1.119 \\
1.212\end{array}$ & $\begin{array}{l}1.366 \\
1.185\end{array}$ & $\begin{array}{l}1.204 \\
1.126\end{array}$ & $\begin{array}{l}1.335 \\
1.347\end{array}$ & $\begin{array}{l}1.420 \\
1.475\end{array}$ & $\begin{array}{l}1.128 \\
1.108\end{array}$ \\
\hline 3 & $\frac{1}{5}$ & $\begin{array}{l}1.037 \\
1.021\end{array}$ & $\begin{array}{l}0.992 \\
0.949\end{array}$ & $\begin{array}{l}1.065 \\
1.078\end{array}$ & $\begin{array}{l}1.164 \\
1.098\end{array}$ & $\begin{array}{l}1.127 \\
1.219\end{array}$ & $\begin{array}{l}1.062 \\
1.066\end{array}$ \\
\hline 4 & $\frac{1}{5}$ & $\begin{array}{l}0.988 \\
1.011\end{array}$ & $\begin{array}{l}1.070 \\
0.978\end{array}$ & $\begin{array}{l}0.989 \\
1.017\end{array}$ & $\begin{array}{l}1.172 \\
1.162\end{array}$ & $\begin{array}{l}1.192 \\
1.208\end{array}$ & $\begin{array}{l}1.049 \\
1.041\end{array}$ \\
\hline 5 & $\frac{1}{5}$ & $\begin{array}{l}1.109 \\
0.932\end{array}$ & $\begin{array}{l}1.108 \\
0.892\end{array}$ & $\begin{array}{l}1.126 \\
0.957\end{array}$ & $\begin{array}{l}1.136 \\
0.986\end{array}$ & $\begin{array}{l}1.201 \\
1.119\end{array}$ & $\begin{array}{l}1.088 \\
1.097\end{array}$ \\
\hline 6 & $\frac{1}{5}$ & $\begin{array}{l}1.236 \\
1.663\end{array}$ & $\begin{array}{l}1.349 \\
1.446\end{array}$ & $\begin{array}{l}1.406 \\
1.390\end{array}$ & $\begin{array}{l}1.619 \\
1.601\end{array}$ & $\begin{array}{l}1.245 \\
1.271\end{array}$ & $\begin{array}{l}1.117 \\
1.145\end{array}$ \\
\hline 7 & $\begin{array}{l}1 \\
5\end{array}$ & $\begin{array}{l}1.216 \\
1.246\end{array}$ & $\begin{array}{l}1.253 \\
1.257\end{array}$ & $\begin{array}{l}1.321 \\
1.328\end{array}$ & $\begin{array}{l}1.372 \\
1.663\end{array}$ & $\begin{array}{l}1.877 \\
1.902\end{array}$ & $\begin{array}{l}1.194 \\
1.182\end{array}$ \\
\hline 8 & $\frac{1}{5}$ & $\begin{array}{l}1.214 \\
1.246\end{array}$ & $\begin{array}{l}1.253 \\
1.257\end{array}$ & $\begin{array}{l}1.321 \\
1.329\end{array}$ & $\begin{array}{l}1.372 \\
1.663\end{array}$ & $\begin{array}{l}2.271 \\
2.412\end{array}$ & $\begin{array}{l}1.226 \\
1.279\end{array}$ \\
\hline 9 & $\begin{array}{l}\frac{1}{5} \\
\text {. }\end{array}$ & $\begin{array}{l}0.994 \\
0.987\end{array}$ & $\begin{array}{l}1.226 \\
1.051\end{array}$ & $\begin{array}{l}1.181 \\
1.200\end{array}$ & $\begin{array}{l}1.467 \\
1.584\end{array}$ & $\begin{array}{l}1.304 \\
1.272\end{array}$ & $\begin{array}{l}1.101 \\
1.117\end{array}$ \\
\hline 10 & $\frac{1}{5}$ & $\begin{array}{l}1.024 \\
0.960\end{array}$ & $\begin{array}{l}1.180 \\
1.100\end{array}$ & $\begin{array}{l}1.224 \\
1.191\end{array}$ & $\begin{array}{l}1.555 \\
1.676\end{array}$ & $\begin{array}{l}1.122 \\
1.008\end{array}$ & $\begin{array}{l}1.110 \\
1.119\end{array}$ \\
\hline 11 & $\frac{1}{5}$ & $\begin{array}{l}1.346 \\
1.194\end{array}$ & $\begin{array}{l}0.954 \\
1.015\end{array}$ & $\begin{array}{l}1.511 \\
1.147\end{array}$ & $\begin{array}{l}1.117 \\
1.101\end{array}$ & $\begin{array}{l}1.084 \\
0.997\end{array}$ & $\begin{array}{l}1.090 \\
1.108\end{array}$ \\
\hline 12 & $\frac{1}{5}$ & $\begin{array}{l}1.028 \\
0.955\end{array}$ & $\begin{array}{l}0.964 \\
0.863\end{array}$ & $\begin{array}{l}1.173 \\
1.509\end{array}$ & $\begin{array}{l}1.895 \\
1.801\end{array}$ & $\begin{array}{l}1.994 \\
1.867\end{array}$ & $\begin{array}{l}1.128 \\
1.148\end{array}$ \\
\hline 13 & $\frac{1}{5}$ & $\begin{array}{l}1.357 \\
1.425\end{array}$ & $\begin{array}{l}1.010 \\
0.990\end{array}$ & $\begin{array}{l}1.371 \\
1.547\end{array}$ & $\begin{array}{l}1.189 \\
1.138\end{array}$ & $\begin{array}{l}1.277 \\
1.257\end{array}$ & $\begin{array}{l}1.089 \\
1.102\end{array}$ \\
\hline 14 & $\frac{1}{5}$ & $\begin{array}{l}1.216 \\
1.143\end{array}$ & $\begin{array}{l}1.087 \\
0.980\end{array}$ & $\begin{array}{l}1.318 \\
1.153\end{array}$ & $\begin{array}{l}1.418 \\
1.375\end{array}$ & $\begin{array}{l}1.322 \\
1.307\end{array}$ & $\begin{array}{l}1.166 \\
1.109\end{array}$ \\
\hline 15 & $\frac{1}{5}$ & $\begin{array}{l}1.461 \\
1.534\end{array}$ & $\begin{array}{l}1.230 \\
1.241\end{array}$ & $\begin{array}{l}1.687 \\
2.077\end{array}$ & $\begin{array}{l}1.402 \\
1.702\end{array}$ & $\begin{array}{l}2.527 \\
2.602\end{array}$ & $\begin{array}{l}1.161 \\
1.125\end{array}$ \\
\hline$A V E$ & $\frac{1}{5}$ & $\begin{array}{l}1.167 \\
1.189\end{array}$ & $\begin{array}{l}1.161 \\
1.105\end{array}$ & $\begin{array}{l}1.277 \\
1.294\end{array}$ & $\begin{array}{l}1.375 \\
1.422\end{array}$ & $\begin{array}{l}1.496 \\
1.495\end{array}$ & $\begin{array}{l}1.129 \\
1.126\end{array}$ \\
\hline
\end{tabular}

Notes: PBK and PBUK represent the post-break method with the known and unknown break respectively. NVK and NVUK represent the Naive ARFIMA-based method with known and unknown break respectively. NOVO represents the $\operatorname{ARFIMA}\left(d^{*}\right)$ based method and ARFA means the adapative AR forecast method. AVE means the average of RMSFEs. 
Table 4. Relative Root Mean Squared Forecast Error (Rolling Window)

\begin{tabular}{cccccccc}
\hline \hline$E X$ & $h$ & $P B K$ & $N V K$ & $P B U K$ & $N V U K$ & $N V N O$ & $A F A R$ \\
\hline 1 & 1 & 1.024 & 1.207 & 1.151 & 1.372 & 1.466 & 1.122 \\
& 5 & 1.018 & 1.230 & 1.212 & 1.286 & 1.435 & 1.129 \\
& 1 & 1.065 & 1.249 & 1.108 & 1.226 & 1.407 & 1.110 \\
3 & 5 & 1.091 & 1.221 & 1.002 & 1.247 & 1.426 & 1.092 \\
& 1 & 1.057 & 1.108 & 1.085 & 1.204 & 1.086 & 1.046 \\
4 & 1 & 1.018 & 0.931 & 1.102 & 1.118 & 1.054 & 1.048 \\
& 5 & 1.998 & 1.050 & 1.100 & 1.202 & 1.077 & 1.030 \\
5 & 1 & 1.155 & 1.104 & 1.027 & 1.182 & 1.046 & 1.036 \\
& 5 & 0.966 & 0.992 & 0.969 & 1.144 & 1.098 & 1.075 \\
6 & 1 & 1.021 & 1.119 & 1.217 & 1.339 & 1.070 & 1.066 \\
& 5 & 0.993 & 1.046 & 1.090 & 1.201 & 1.174 & 1.120 \\
7 & 1 & 1.154 & 1.133 & 1.219 & 1.272 & 1.621 & 1.152 \\
& 5 & 1.198 & 1.134 & 1.228 & 1.163 & 1.602 & 1.182 \\
& 1 & 1.204 & 1.231 & 1.228 & 1.302 & 1.982 & 1.198 \\
& 5 & 1.162 & 1.229 & 1.202 & 1.321 & 1.902 & 1.180 \\
& 1 & 0.992 & 1.265 & 1.198 & 1.512 & 1.205 & 1.054 \\
& 5 & 0.971 & 1.167 & 1.201 & 1.574 & 1.172 & 1.067 \\
& 1 & 1.074 & 1.176 & 1.281 & 1.585 & 1.088 & 1.078 \\
& 5 & 1.002 & 1.116 & 1.297 & 1.606 & 1.001 & 1.060 \\
11 & 1 & 1.431 & 1.014 & 1.539 & 1.318 & 1.037 & 1.082 \\
& 5 & 1.302 & 1.016 & 1.217 & 1.265 & 0.989 & 1.112 \\
12 & 1 & 1.112 & 0.934 & 1.063 & 1.783 & 1.509 & 1.138 \\
& 5 & 0.962 & 0.822 & 1.406 & 1.393 & 1.487 & 1.166 \\
13 & 1 & 1.369 & 1.019 & 1.307 & 1.229 & 1.201 & 1.084 \\
& 5 & 1.452 & 1.000 & 1.516 & 1.297 & 1.194 & 1.097 \\
14 & 1 & 1.126 & 1.057 & 1.198 & 1.196 & 1.262 & 1.121 \\
& 5 & 0.993 & 0.940 & 1.076 & 1.210 & 1.216 & 1.156 \\
& 1 & 1.341 & 1.236 & 1.881 & 1.359 & 2.217 & 1.137 \\
& 5 & 1.389 & 1.313 & 1.918 & 1.476 & 2.172 & 1.176 \\
\hline \hline
\end{tabular}


Table 5. Forecast Evaluation of $\eta_{T+1}$

\begin{tabular}{|c|c|c|c|c|c|c|}
\hline $\mathrm{EX}$ & & $\alpha_{0}$ & $\alpha_{1}$ & $\alpha_{2}$ & $R^{2}$ & $P(F)$ \\
\hline \multirow[t]{7}{*}{1} & AR & $0.010(0.054)$ & $0.957(0.090)$ & & 0.266 & \\
\hline & $\mathrm{AR}+\mathrm{PBK}$ & $-0.022(0.079)$ & $0.890(0.132)$ & $0.169(0.100)$ & & 0.38 \\
\hline & $\mathrm{AR}+\mathrm{PBUK}$ & $0.039(0.087)$ & $0.927(0.127)$ & $0.102(0.136)$ & 0.268 & 0.49 \\
\hline & $\mathrm{AR}+\mathrm{NVK}$ & $0.018(0.049)$ & $0.900(0.099)$ & $0.158(0.104)$ & 0.281 & 0.40 \\
\hline & $\mathrm{AR}+\mathrm{NVUK}$ & $0.044(0.076)$ & $0.918(0.116)$ & $0.118(0.167)$ & 0.267 & 0.44 \\
\hline & $\mathrm{AR}+\mathrm{NVNO}$ & $-0.107(0.129)$ & $0.937(0.136)$ & $0.087(0.126)$ & 0.266 & 0.59 \\
\hline & $\mathrm{AR}+\mathrm{AFAR}$ & $-0.014(0.090)$ & $0.857(0.147)$ & $0.246(0.142)$ & & 0.26 \\
\hline \multirow[t]{7}{*}{2} & AR & $0.021(0.049)$ & $0.927(0.110)$ & & 0.255 & \\
\hline & $\mathrm{AR}+\mathrm{PBK}$ & $-0.032(0.103)$ & $0.897(0.105)$ & $0.139(0.174)$ & 0.276 & 0.41 \\
\hline & $\mathrm{AR}+\mathrm{PBUK}$ & $0.049(0.095)$ & $0.908(0.119)$ & $0.124(0.201)$ & 0.257 & 0.45 \\
\hline & $\mathrm{AR}+\mathrm{NVK}$ & $0.027(0.117)$ & $0.901(0.119)$ & $0.147(0.202)$ & 0.266 & 0.44 \\
\hline & $\mathrm{AR}+\mathrm{NVUK}$ & $0.097(0.187)$ & $0.927(0.100)$ & $0.109(0.216)$ & 0.259 & 0.52 \\
\hline & $\mathrm{AR}+\mathrm{NVNO}$ & $-0.156(0.190)$ & $0.912(0.187)$ & $0.104(0.232)$ & & 0.48 \\
\hline & $\mathrm{AR}+\mathrm{AFAR}$ & $-0.075(0.102)$ & $0.887(0.169)$ & $0.194(0.168)$ & 0.278 & 0.39 \\
\hline \multirow[t]{7}{*}{4} & $\mathrm{AR}$ & $0.017(0.049)$ & $0.978(0.103)$ & & 0.353 & \\
\hline & $\mathrm{AR}+$ & & & & & 0.32 \\
\hline & $\mathrm{AR}+\mathrm{PBUK}$ & $0.062(0.092)$ & $0.809(0.148)$ & $0.176(0.148)$ & & 0.36 \\
\hline & & $0.038(0.064)$ & $0.857(0.132)$ & $0.211(0.191)$ & 0.360 & 0.34 \\
\hline & $\mathrm{AR}+\mathrm{NVUK}$ & $0.089(0.112)$ & $0.882(0.147)$ & $0.199(0.241)$ & 0.354 & 0.36 \\
\hline & $\mathrm{AR}+\mathrm{NVNO}$ & $-0.022(0.054)$ & $0.876(0.129)$ & $0.201(0.154)$ & 0.358 & 0.34 \\
\hline & $\mathrm{AR}+\mathrm{AFAR}$ & $-0.021(0.090)$ & $0.789(0.156)$ & $0.344(0.239)$ & 0.386 & 0.20 \\
\hline \multirow[t]{7}{*}{6} & $\mathrm{AR}(\mathrm{k})$ & $-0.052(0.067)$ & $0.928(0.103)$ & & 0.310 & \\
\hline & $\mathrm{AR}+\mathrm{PBK}$ & $0.027(0.085)$ & $0.869(0.125)$ & $0.157(0.217)$ & 0.330 & 0.44 \\
\hline & $\mathrm{AR}+\mathrm{PBUK}$ & $0.093(0.101)$ & $0.901(0.156)$ & $0.103(0.165)$ & 0.319 & 0.49 \\
\hline & $\mathrm{AR}+\mathrm{NVK}$ & $-0.049(0.076)$ & $0.879(0.117)$ & $0.201(0.189)$ & 0.326 & 0.45 \\
\hline & $\mathrm{AR}+\mathrm{NVUK}$ & $-0.088(0.101)$ & $0.889(0.137)$ & $0.169(0.217)$ & 0.310 & 0.50 \\
\hline & $\mathrm{AR}+\mathrm{NVNO}$ & $0.100(0.079)$ & $0.899(0.119)$ & $0.108(0.134)$ & 0.312 & 0.49 \\
\hline & $\mathrm{AR}+\mathrm{AFAR}$ & $-0.029(0.149)$ & $0.801(0.145)$ & $0.349(0.289)$ & 0.341 & 0.40 \\
\hline \multirow[t]{7}{*}{7} & & $0.032(0.096)$ & $0.936(0.109)$ & & 0.322 & \\
\hline & $\mathrm{AR}+\mathrm{PBK}$ & $0.048(0.133)$ & $0.902(0.155)$ & $0.166(0.184)$ & 0.332 & 0.53 \\
\hline & $\mathrm{AR}+\mathrm{PBUK}$ & $0.078(0.141)$ & $0.908(0.177)$ & $0.141(0.201)$ & 0.328 & 0.58 \\
\hline & $\mathrm{AR}+\mathrm{NVK}$ & $0.039(0.093)$ & $0.907(0.185)$ & $0.199(0.169)$ & 0.329 & 0.56 \\
\hline & NVUK & $0.109(0.101)$ & $0.889(0.189)$ & $0.121(0.176)$ & 0.325 & 0.60 \\
\hline & JVNO & $-0.209(0.232)$ & $1.044(0.129)$ & $0.102(0.279)$ & 0.322 & 0.64 \\
\hline & $\mathrm{AR}+\mathrm{AFAR}$ & $-0.011(0.097)$ & $0.849(0.134)$ & $0.243(0.203)$ & 0.352 & 0.46 \\
\hline
\end{tabular}


Table 5. Continued

\begin{tabular}{|c|c|c|c|c|c|c|}
\hline EX & $A M$ & $\alpha_{0}$ & $\alpha_{1}$ & $\alpha_{2}$ & $R^{2}$ & $P(F)$ \\
\hline \multirow[t]{7}{*}{8} & $\mathrm{AR}(\mathrm{k})$ & $-0.092(0.111)$ & $1.100(0.123)$ & & 0.296 & \\
\hline & $\mathrm{AR}+\mathrm{PBK}$ & $0.094(0.109)$ & $0.942(0.169)$ & $0.141(0.113)$ & 0.320 & 0.49 \\
\hline & $\mathrm{AR}+\mathrm{PBUK}$ & $0.108(0.153)$ & $0.877(0.173)$ & $0.202(0.186)$ & 0.306 & 0.58 \\
\hline & $\mathrm{AR}+\mathrm{NVK}$ & $0.100(0.121)$ & $0.902(0.159)$ & $0.167(0.173)$ & 0.314 & 0.50 \\
\hline & $\mathrm{AR}+\mathrm{NVUK}$ & $0.126(0.123)$ & $0.860(0.181)$ & $0.190(0.202)$ & 0.300 & 0.61 \\
\hline & $\mathrm{AR}+\mathrm{NVNO}$ & $-0.185(0.196)$ & $1.022(0.129)$ & $0.109(0.279)$ & 0.305 & 0.58 \\
\hline & $\mathrm{AR}+\mathrm{AFAR}$ & $-0.096(0.165)$ & $0.889(0.104)$ & $0.161(0.224)$ & 0.318 & 0.52 \\
\hline \multirow[t]{7}{*}{10} & $\mathrm{AR}$ & $0.031(0.082)$ & $0.932(0.123)$ & & 0.346 & \\
\hline & $\mathrm{AR}+\mathrm{PBK}$ & $0.113(0.108)$ & $0.869(0.167)$ & & 362 & 0.34 \\
\hline & $\mathrm{AR}+\mathrm{PBUk}$ & $0.141(0.137)$ & $0.841(0.139)$ & $0.241(0.204)$ & 0.352 & 0.39 \\
\hline & $\mathrm{AR}+\mathrm{NVK}$ & $0.126(0.143)$ & $0.842(0.135)$ & $0.214(0.210)$ & 0.358 & 0.37 \\
\hline & $\mathrm{AR}+\mathrm{NVUK}$ & $0.152(0.167)$ & $0.887(0.187)$ & $0.165(0.167)$ & 0.349 & 0.42 \\
\hline & $\mathrm{AR}+\mathrm{NVNO}$ & $-0.029(0.119)$ & $0.857(0.091)$ & $0.201(0.201)$ & 0.359 & 0.37 \\
\hline & $\mathrm{AR}+\mathrm{AFAR}$ & $0.045(0.135)$ & $0.821(0.114)$ & $0.333(0.199)$ & 0.376 & 0.28 \\
\hline \multirow[t]{7}{*}{12} & $\mathrm{AR}$ & $-0.101(0.177)$ & $0.878(0.089)$ & & 0.259 & \\
\hline & $\mathrm{AR}+\mathrm{PBK}$ & $0.141(0.123)$ & $0.802(0.177)$ & $0.371(0.194)$ & 0.271 & 0.63 \\
\hline & $\mathrm{AR}+\mathrm{PBUK}$ & $0.177(0.157)$ & $0.866(0.119)$ & $0.207(0.186)$ & 0.263 & 0.68 \\
\hline & $\mathrm{AR}+\mathrm{NV}$ & $0.102(0.129)$ & $0.849(0.165)$ & $0.254(0.201)$ & 0.278 & 0.60 \\
\hline & $\mathrm{AR}+\mathrm{NVUK}$ & $-0.196(0.178)$ & $0.860(0.179)$ & $0.288(0.171)$ & 0.263 & 0.68 \\
\hline & $\mathrm{AR}+\mathrm{NVNO}$ & $0.149(0.281)$ & $0.822(0.129)$ & $0.171(0.288)$ & 0.265 & 0.69 \\
\hline & $\mathrm{AR}+\mathrm{AFAR}$ & $-0.103(0.129)$ & $0.843(0.192)$ & $0.298(0.219)$ & 0.284 & 0.55 \\
\hline
\end{tabular}

Table 6. Root Mean Squared Forecast Error Value of $\eta_{T_{1}+1}$

\begin{tabular}{cccccc}
\hline \hline Experiments & $h=1$ & $h=2$ & $h=3$ & $h=4$ & $h=5$ \\
\hline AR & & & & & \\
6 & 1.0092 & 1.0703 & 1.1096 & 1.1702 & 1.1813 \\
8 & 1.0227 & 1.0295 & 1.0546 & 1.0909 & 1.0887 \\
12 & 1.0095 & 1.0211 & 1.0842 & 1.1293 & 1.1403 \\
NVNO & & & & & \\
6 & 1.8350 & 1.7950 & 1.7175 & 1.6747 & 1.7030 \\
8 & 1.9345 & 2.0178 & 2.1541 & 2.1919 & 2.2887 \\
12 & 1.0175 & 1.0241 & 1.1842 & 1.1593 & 1.2403 \\
AFAR & & & & & \\
6 & 1.1068 & 1.0624 & 1.1112 & 1.1628 & 1.1800 \\
8 & 1.0369 & 1.0478 & 1.0591 & 2.1221 & 1.1276 \\
12 & 1.1075 & 1.0281 & 1.1139 & 1.1333 & 1.1479 \\
\hline \hline
\end{tabular}


Table 7. Statistics for the Log-Realized Volatilities

\begin{tabular}{cccc}
\hline \hline Statistic & $D E M / U S D$ & USD/YEN & GBP/USD \\
\hline $\mathrm{T}$ & 3056 & 3071 & \\
Mean & -0.99951 & -0.87484 & 3063 \\
Std.Devn & 0.7165 & 0.80617 & -1.0810 \\
Skewness & 0.3198 & 0.2520 & 0.6799 \\
Excess Kurtosis & 0.5255 & 0.5606 & 0.3775 \\
Normality $\chi^{2}(2)$ & 55.729 & 47.559 & 69.825 \\
Minimum & -3.3947 & -3.5447 & -3.1705 \\
Maximum & 1.8967 & 3.6103 & 2.0873 \\
\hline \hline
\end{tabular}

Table 8. Number of Breaks and Break Dates

\begin{tabular}{ccccccc}
\hline \hline Series & $\#$ & $\mathrm{~T}_{1}$ & $\mathrm{~T}_{2}$ & $\mathrm{~T}_{3}$ & $\mathrm{~T}_{4}$ & $\mathrm{~T}_{5}$ \\
\hline DEM/USD & 5 & 1989.5 .22 & 1991.3 .21 & 1993.3 .11 & 1995.9 .1 & 1997.7 .2 \\
USD/YEN & 5 & 1989.5 .22 & 1991.7 .8 & 1993.4 .20 & 1997.6 .30 & 1998.7 .20 \\
GBP/USD & 4 & 1989.5 .19 & 1991.4 .3 & 1995.8 .25 & 1997.7 .1 & \\
\hline \hline
\end{tabular}

Table 9. Relative Root Mean Squared Forecast Error Values

\begin{tabular}{ccrccccc}
\hline \hline Series & $h$ & $P B$ & $N V$ & NVNO & HAR & VRB & AFAR \\
\hline DEM & 1 & 1.586 & 1.487 & 1.551 & 1.068 & 1.214 & 1.093 \\
& 3 & 1.578 & 1.502 & 1.526 & 1.062 & 1.208 & 1.108 \\
YEN & 1 & 1.412 & 1.689 & 1.231 & 1.046 & 1.181 & 1.063 \\
& 3 & 1.398 & 1.668 & 1.256 & 1.054 & 1.186 & 1.098 \\
GBP & 1 & 1.566 & 1.595 & 1.519 & 1.032 & 1.163 & 1.060 \\
& 3 & 1.612 & 1.584 & 1.511 & 1.030 & 1.158 & 1.047 \\
\hline \hline
\end{tabular}


Table 10. Out-of-Sample Forecast Evaluation

\begin{tabular}{|c|c|c|c|c|c|c|}
\hline EX & & $\alpha_{0}$ & $\alpha_{1}$ & $\alpha_{2}$ & $R^{2}$ & $P(F)$ \\
\hline \multirow[t]{7}{*}{ DEM } & $\mathrm{AR}$ & $0.088(0.074)$ & $0.918(0.129)$ & & 0.338 & \\
\hline & $\mathrm{AR}+\mathrm{PB}$ & $0.112(0.189)$ & $0.900(0.172)$ & $0.179(0.130)$ & 0.340 & 0.62 \\
\hline & $\mathrm{AR}+\mathrm{NV}$ & $-0.089(0.053)$ & $0.887(0.190)$ & $0.218(0.152)$ & 0.345 & 0.52 \\
\hline & $\mathrm{AR}+\mathrm{NVNO}$ & $-0.118(0.109)$ & $0.863(0.129)$ & $0.243(0.134)$ & 0.344 & 0.44 \\
\hline & $\mathrm{AR}+\mathrm{HAR}$ & $-0.038(0.089)$ & $0.805(0.119)$ & $0.292(0.097)$ & 0.373 & 0.24 \\
\hline & $\mathrm{AR}+\mathrm{VRB}$ & $-0.091(0.165)$ & $0.842(0.131)$ & $0.263(0.126)$ & 0.356 & 0.36 \\
\hline & $\mathrm{AR}+\mathrm{AFAR}$ & $-0.069(0.190)$ & $0.798(0.149)$ & $0.337(0.219)$ & 0.377 & 0.26 \\
\hline \multirow[t]{7}{*}{ YEN } & $\mathrm{AR}$ & $0.029(0.089)$ & $0.945(0.129)$ & & 0.403 & \\
\hline & $\mathrm{AR}+\mathrm{PB}$ & $-0.068(0.116)$ & $0.872(0.112)$ & $0.216(0.174)$ & 0.407 & 0.46 \\
\hline & $\mathrm{AR}+\mathrm{NV}$ & $-0.063(0.105)$ & $0.890(0.109)$ & $0.200(0.168)$ & 0.408 & 0.48 \\
\hline & $\mathrm{AR}+\mathrm{NVNO}$ & $-0.056(0.111)$ & $0.851(0.122)$ & $0.281(0.154)$ & 0.414 & 0.36 \\
\hline & $\mathrm{AR}+\mathrm{HAR}$ & $0.020(0.097)$ & $0.775(0.190)$ & $0.337(0.201)$ & 0.445 & 0.20 \\
\hline & $\mathrm{AR}+\mathrm{VRB}$ & $0.046(0.110)$ & $0.836(0.157)$ & $0.289(0.232)$ & 0.423 & 0.26 \\
\hline & $\mathrm{AR}+\mathrm{AFAR}$ & $-0.049(0.127)$ & $0.801(0.149)$ & $0.321(0.219)$ & 0.377 & 0.24 \\
\hline \multirow[t]{7}{*}{ GBP } & $\mathrm{AR}$ & $0.048(0.094)$ & $0.906(0.145)$ & & 0.364 & \\
\hline & $\mathrm{AR}+\mathrm{PB}$ & $-0.090(0.107)$ & $0.892(0.175)$ & $0.199(0.134)$ & 0.370 & 0.59 \\
\hline & $\mathrm{AR}+\mathrm{NV}$ & $-0.123(0.156)$ & $0.879(0.148)$ & $0.169(0.108)$ & 0.367 & 0.54 \\
\hline & $\mathrm{AR}+\mathrm{NVNO}$ & $-0.138(0.184)$ & $0.860(0.168)$ & $0.234(0.178)$ & 0.368 & 0.47 \\
\hline & $\mathrm{AR}+\mathrm{HAR}$ & $-0.028(0.192)$ & $0.765(0.179)$ & $0.361(0.214)$ & 0.379 & 0.22 \\
\hline & $\mathrm{AR}+\mathrm{VRB}$ & $-0.061(0.104)$ & $0.883(0.158)$ & $0.257(0.140)$ & 0.373 & 0.33 \\
\hline & $\mathrm{AR}+\mathrm{AFAR}$ & $-0.027(0.036)$ & $0.801(0.169)$ & $0.304(0.231)$ & 0.386 & 0.18 \\
\hline
\end{tabular}

\section{Acknowledgments}

We would like to thank Harrison Cheng for helpful decision. Cheng Hsiao wishes to thank the NSF of China grant \# 71131008 for partial support of this research. Bauwens and Wang were supported by the contract "Projet d'Actions de Recherche Concertées" 07/12-002 of the "Communauté francaise de Belgique", granted by the "Académie universitaire Louvain". We also thank the referees and the editors, as well as the seminar participants at CORE, University of Aarhus, the 2011 conference in Honor of Professor Hashem Pesaran and the 32th International Symposium on Forecasting for insightful suggestions and comments.

\section{References}

Andersen, T.G., T. Bollerslev, F.X. Diebold and P. Labys (2001): " The Distribution of Realized Exchange Rate Volatility," Journal of the American Statistical Association, 96, 42-55. 
Andersen, T.G., T. Bollerslev, F.X. Diebold and P. Labys (2003): " Modeling and Forecasting Realized Volatility, " Econometrica, 71, 529-626.

Berk, K. N. (1974): "Consistent Autoregressive Spectral Sstimates, " Ann. Statist. 2, 489-502.

Brockwell, P. J. and R. A. Davis (1991): Time Series: Theory and Methods, 2nd Edn. New York: Springer-Verlag.

Bai, J. and P. Perron (1998): "Estimating and Testing Linear Models with Multiple Structural Changes, " Econometrica, 66, 47-78.

Baillie, R. T.(1996): " Long Memory Processes and Fractional Integration in Econometrics, " Journal of Econometrics 73, 5-59.

Baillie, R. T. and C. Morana (2009): " Modeling Long Memory and Structural Breaks in Conditional Variances: an Adaptive FIGARCH Approach, " Journal of Economic Dynamics and Control, 33, 1577-1592.

Beran, J. and N. Terrin. 1996. "Testing for a change of the long-memory parameter," Biometrika, 83, 627-638.

Bollerslev, T. and H. O. Mikkelsen (1996):" Modeling and Pricing Long memory In Stock Market Volatility, " Journal of Econometrics, 73, 151-184.

Clements, M.P. and D.F. Hendry (1998): "Forecasting Economic Time Series". Cambridge University Press, Cambridge.

Choi, K., Yu, W.C., Zivot, E., (2010): " Long Memory versus Structural Breaks in Modeling and forecasting Realized Volatility", Journal of International Money and Finance, 29, 857-875.

Crator, N. and Ray, B. (1996): " Model Selection and Forecasting for Long-Range Dependent Processes," Journal of Forecasting, 15, 107-125.

Ding, Z., Granger, C. W. J. and Engle, R. F. (1993): " A Long Memory Property of Stock Mark Returns and a New Model," Journal of Empirical Finance 1, 83-106.

Diebold, F. X. and A. Inoue (2001): “Long Memory and Regime Switching," Journal of Econometrics 105, 131-159.

Granger, C.W.J., (1980): "Long Memory Relationships and the Aggregation of Dynamic Models," Journal of Econometrics 14, 227-238.

Granger, C.W.J. and N. Hyung (2004): "Occasional Structural Breaks and Long Memory with an Application to the S\&P 500 Absolute Stock Returns. " Journal of Empirical Finance, 11, 399-421. 
Hosking, J. R. M. (1996): "Asymptotic Distributions of the Sample Mean, Autocovariances, and Autocorrelations of Long-Memory Time Series." Journal of Econometrics 73, 261-284.

Hyung, N. and Franses, P. (2001): " Structural breaks and long memory in US inflation rates: Do they matter for forecasting?" Econometric Institute Research Report, EI-2001-13.

Hansen, P.H. and A. Timmermann (2012): " Choice of Sample Split in Out-of-Sample Forecast Evaluation. " EUI Working Paper ECO 2012/10.

Kuan, C.-M. and C.-C. Hsu (1998): " Change-Point Estimation of Fractionally Integrated Processes." Journal of Time Series Analysis 19, 693-708.

Mallows, C. L. (1973): “Come Comments on $C_{p}$," Technometrics, 15, 661-675.

Mincer, J. and V. Zarnowitz. (1969): " The Evaluation of Economic Forecasts, " in Economic Forecasts and Expectations, edited by W.F.Sharpe and C.M. Cooter. New Jersey: Prentice-Hall.

Nunes, L. C., C.-M. Kuan and P. Newbold (1995): " Spurious break." Econometric Theory 11, 736-749.

Pesaran, M.H. and A. Timmermann (2005): "Small Sample Properties of Forecasts from Autoregressive Models under Structural Breaks, " Journal of Econometrics $129,183-217$.

Pesaran, M.H. and A. Timmermann (2007): "Selection of Estimation Window in the Presence of breaks, " Journal of Econometrics 129, 183-217.

Stock, J. H. and M. W. Watson (1996): " Evidence on Structural Instability in Macroeconomic time Series Realtions." Journal of Business and Economics Statistics 14, 11-30.

Tiao, G. C. nd R. S. Tsay (1994): " Some Advances in Non Linear and Adaptive Modelling in Time Series." Journal of Forecasting 13(2), 109-148.

Wang, S. H. and C. Hsiao (2012): " Testing Uncorrelatedness Between Two Memory Processes. "3rd Revision .

\section{APPENDIX 9}

\section{A.1. Proof of Lemma 1}

We follow Granger (1980) and Diebold and Inoue (2001) to define a process $\eta_{t}$ as long memory if the rate of growth of variances of partial sums $\operatorname{Var}\left(S_{T}\right)=O\left(T^{2 d+1}\right)$, where $-0.5<d<0.5, d \neq 0$, and $S_{T}=\sum_{t=1}^{T} \eta_{t}$.

9 The detailed proofs are available on request. 
By Theorem 13.2.1 of Brockwell and Davis (1991), we know that

$$
\eta_{t}=\mu_{1}+(1-L)^{-d_{1}} e_{t}=\mu_{1}+\sum_{v=0}^{\infty} \psi_{v} e_{t-v}, \quad E \eta_{t}=\mu_{1}, \quad \text { as } \quad 1 \leq t \leq T_{1},
$$

and $\quad \eta_{t}=\mu_{2}+(1-L)^{-d_{2}} e_{t}=\mu_{2}+\sum_{s=0}^{\infty} \psi_{s}^{*} e_{t-s}, \quad E \eta_{t}=\mu_{2}, \quad$ as $\quad T_{1}+1 \leq t \leq T_{2}$ where $\psi_{v} \sim v^{d_{1}-1}$ and $\psi_{s}^{*} \sim s^{d_{2}-1}$.

$$
\begin{aligned}
& \operatorname{Var}\left(\sum_{t=1}^{T} \eta_{t}\right)=\operatorname{Var}\left(\sum_{t=1}^{T_{1}} \eta_{t}+\sum_{t=T_{1}+1}^{T} \eta_{t}\right)=\operatorname{Var}\left(\sum_{t=1}^{T_{1}} \eta_{t}\right)+\operatorname{Var}\left(\sum_{t=T_{1}+1}^{T} \eta_{t}\right) \\
& +2 \sum_{t=1}^{T_{1}} \sum_{s=T_{1}+1}^{T} \operatorname{Cov}\left(\eta_{t}, \eta_{s}\right)=(A)+(B)+(C) .
\end{aligned}
$$

To prove the asymptotic property of (A.1), we investigate the property of (A) on the right hand side of (A.1). We first let $t-v=h$ and note that

$$
\begin{aligned}
& (A)=\operatorname{Var}\left(\sum_{t=1}^{T_{1}}\left(\mu_{1}+\sum_{v=0}^{\infty} \psi_{v} e_{t-v}\right)\right)=\operatorname{Var}\left(\sum_{t=1}^{T_{1}} \sum_{v=0}^{\infty} \psi_{v} e_{t-v}\right)=E\left(\sum_{t=1}^{T_{1}} \sum_{v=0}^{\infty} \psi_{v} e_{t-v}\right)^{2} \\
& =\sum_{t=1}^{T_{1}} E\left(\sum_{v=0}^{\infty} \psi_{v} e_{t-v}\right)^{2}+\sum_{t=1}^{T_{1}-1} \sum_{s=t+1}^{T_{1}} E\left(\left(\sum_{v=0}^{\infty} \psi_{v} e_{t-v}\right)\left(\sum_{v=0}^{\infty} \psi_{v} e_{s-v}\right)\right) \\
& =O\left(T_{1}^{2 d_{1}+1}\right)=O\left(\kappa^{2 d_{1}+1} T^{2 d_{1}+1}\right) .
\end{aligned}
$$

Similarly, it follows that

$$
(B)=\operatorname{Var}\left(\sum_{t=T_{1}+1}^{T} \eta_{t}\right)=O_{p}\left(T_{2}^{2 d_{2}+1}\right)=O\left((1-\kappa)^{2 d_{2}+1} T^{2 d_{2}+1}\right) .
$$

Let $s^{\prime}=s / T$ and $t^{\prime}=t / T$, we further show that

$$
\begin{aligned}
& \sum_{t=1}^{T_{1}} \sum_{s=T_{1}+1}^{T} \operatorname{Cov}\left(\eta_{t}, \eta_{s}\right)=\sum_{t=1}^{T_{1}} \sum_{s=T_{1}+1}^{T} \operatorname{Cov}\left(\mu_{1}+\sum_{v=0}^{\infty} \psi_{v} e_{t-v}, \mu_{2}+\sum_{l=0}^{\infty} \psi_{l}^{*} e_{s-l}\right) \\
& =\sum_{t=1}^{T_{1}} \sum_{s=T_{1}+1}^{T} \operatorname{Cov}\left(\sum_{v=0}^{\infty} \psi_{v} e_{t-v}, \sum_{l=0}^{\infty} \psi_{l}^{*} e_{s-l}\right)=\sum_{t=1}^{T_{1}} \sum_{s=T_{1}+1}^{T} E\left(\left(\sum_{v=0}^{\infty} \psi_{v} e_{t-v}\right)\left(\sum_{l=0}^{\infty} \psi_{l}^{*} e_{s-l}\right)\right) \\
& =\sum_{t=1}^{T_{1}} \sum_{s=T_{1}+1}^{T} \sum_{v=0}^{\infty} \psi_{(s-t)+v} \psi_{v}^{*} \sigma^{2} \sim \sigma^{2} \sum_{t=1}^{T_{1}} \sum_{s=T_{1}+1}^{T} O\left((s-t)^{d_{1}+d_{2}-1}\right) \\
& \sim \sigma^{2} T^{d_{1}+d_{2}+1} \int_{0}^{\kappa} \int_{\kappa}^{1}\left(s^{\prime}-t^{\prime}\right)^{d_{1}+d_{2}-1} d s^{\prime} d t^{\prime} \\
& =O\left(T^{d_{1}+d_{2}+1}\left((1-\kappa)^{d_{1}+d_{2}+1}-\kappa^{d_{1}+d_{2}+1}\right)\right) .
\end{aligned}
$$


It follows from (A.2), (A.3) and (A.4) that

$$
O\left(\operatorname{Var}\left(S_{T}\right)\right)=O\left((\kappa T)^{2 d_{1}+1}+((1-\kappa) T)^{2 d_{2}+1}\right)=O\left(T^{2 d^{*}+1}\right)
$$

where $d^{*}=\lambda d_{1}+(1-\lambda) d_{2}, 0 \leq \lambda \leq 1$ as $T$ is finite. When $T \rightarrow \infty, O\left(\operatorname{Var}\left(S_{T}\right)\right)$ is dominated by $T^{\max \left(d_{1}, d_{2}\right)}$. Therefore, $d^{*} \rightarrow \max \left(d_{1}, d_{2}\right)$.

(A.5) shows that our basic model can be represented by a long memory process with a long memory paramter $d^{*}$ with mean

$$
E\left(\eta_{t}\right)=\kappa \mu_{1}+(1-\kappa) \mu_{2}=\mu^{*}
$$

By (A.5) and (A.6), the Lemma 1 can be established.

\section{A.2. Proof of Theorem 1}

To prove Theorem 1 , by Lemma 1 , we first consider the constant term, $\beta_{0}$, in our AR approximation procedure. From Lemma 1, we have

$$
(1-L)^{d^{*}}\left(\eta_{t}-\mu^{*}\right)=e_{t} \text { and } \quad \eta_{t}=(1-L)^{d^{*}} \mu^{*}+\sum_{j=1}^{\infty} \beta_{j} \eta_{t-j}+e_{t}
$$

where $\beta_{j} \sim C j^{-d^{*}-1}$ and $d^{*} \leq \max \left\{d_{1}, d_{2}\right\}$.

We define $\beta(k)=\left(\beta_{0}, \beta_{1}, \beta_{2}, \ldots, \beta_{k}\right), \quad \beta(k+1, \infty)=\left(\beta_{k+1}, \beta_{k+2}, \ldots,\right)$

$$
\begin{aligned}
\zeta_{k}= & {\left[\begin{array}{ccccc}
1 & \eta_{(k+1)-1} & \eta_{(k+1)-2} & \cdots & \eta_{(k+1)-k} \\
1 & \eta_{(k+2)-1} & \eta_{(k+2)-2} & \cdots & \eta_{(k+2)-k} \\
\vdots & \vdots & \vdots & \vdots & \vdots \\
1 & \eta_{T_{1}-1} & \eta_{T_{2}-2} & \cdots & \eta_{T_{1}-k} \\
\vdots & \vdots & \vdots & \vdots & \vdots \\
1 & \eta_{T-1} & \eta_{T-2} & \cdots & \eta_{T-k}
\end{array}\right]_{(T-k) \times(k+1)}=\left(\widetilde{\eta}_{0}, \widetilde{\eta}_{1}, \widetilde{\eta}_{2}, \ldots, \widetilde{\eta}_{k}\right), } \\
\zeta_{k^{+}}= & {\left[\begin{array}{cccc}
\eta_{(k+1)-(k+1)} & \eta_{(k+1)-(k+2)} & \cdots & \cdots \\
\eta_{(k+2)-(k+1)} & \eta_{(k+2)-(k+2)} & \cdots & \cdots \\
\vdots & \vdots & \vdots & \vdots \\
\eta_{T_{1}-(k+1)} & \eta_{T_{1}-(k+2)} & \cdots & \cdots \\
\vdots & \vdots & \vdots & \vdots \\
\eta_{T-(k+1)} & \eta_{T-2} & \cdots & \cdots
\end{array}\right]=\left(\widetilde{\eta}_{k+1}, \widetilde{\eta}_{k+2}, \ldots,\right), } \\
\varepsilon_{T-k} & =\left(e_{k+1}, e_{T+2}, \cdots, e_{T}\right)^{\prime}, \widetilde{\eta}=\left(\eta_{k+1}, \eta_{k+2}, \ldots, \eta_{T}\right)^{\prime},
\end{aligned}
$$


where $\varepsilon_{T-k}, \widetilde{\eta}$, and all $\widetilde{\eta}_{1}, \widetilde{\eta}_{2}, \cdots, \widetilde{\eta}_{k+1}, \cdots$ are all $(T-k) \times 1$ vectors respectively.

The true model is $\widetilde{\eta}=\zeta_{k} \beta(k)^{\prime}+\zeta_{k^{+}} \beta(k+1, \infty)^{\prime}+\varepsilon_{T-k}$. Because we cannot estimate an infinite number of parameters from a finite sample of $\mathrm{T}$ observations, we then consider the following $k$ th order autoregressive model approximation:

$$
\widetilde{\eta}=\zeta_{k} \beta(k)^{\prime}+\varepsilon_{k},
$$

where $\varepsilon_{k}=\left(e_{k+1, k}, e_{k+2, k}, \cdots, e_{T, k}\right)^{\prime}, e_{t, k}, t=k+1, k+2, \cdots, T$ is the residual derived from fitting $\eta_{t}$ by an $\mathrm{AR}(\mathrm{k})$ approximation.

The adequacy of the approximate model for the true model depends on the choice of $k$. Let $M_{k}=I-\zeta_{k}\left(\zeta_{k}^{\prime} \zeta_{k}\right)^{-1} \zeta_{k}^{\prime}$ be an $(T-k) \times(T-k)$ symmetric, idempotent matrix with the property that $M_{k} \zeta_{k}=0$, then the OLS estimate of the residual of (A.7) $\widehat{\varepsilon}_{k}$ can be written as,

$$
\widehat{\varepsilon}_{k}=M_{k}\left(\varepsilon_{T-k}+\sum_{h=1}^{k} \tilde{\eta}_{h} \beta_{h}+\sum_{h=k+1}^{\infty} \tilde{\eta}_{h} \beta_{h}\right)=M_{k} \zeta_{k^{+}} \beta(k+1, \infty)^{\prime}+M_{k} \varepsilon_{T-k} .
$$

We wish to show that when $-0.5<d^{*}<0.5$,

$$
\begin{aligned}
\widehat{Q} & =T^{-1} \widehat{\varepsilon}_{k}^{\prime} \widehat{\varepsilon}_{k}=T^{-1}\left(\beta(k+1, \infty) \zeta_{k^{+}}^{\prime} M_{k} \zeta_{k^{+}} \beta(k+1, \infty)^{\prime}\right. \\
& \left.+\beta(k+1, \infty) \zeta_{k^{+}}^{\prime} M_{k} \varepsilon_{T-k}+\varepsilon_{T-k}^{\prime} M_{k} \zeta_{k^{+}} \beta(k+1, \infty)^{\prime}+\varepsilon_{T-k}^{\prime} M_{k} \varepsilon_{T-k}\right) \\
& =T^{-1} \beta(k+1, \infty) \zeta_{k^{+}}^{\prime} M_{k} \zeta_{k^{+}} \beta(k+1, \infty)^{\prime}+T^{-1} \beta(k+1, \infty) \zeta_{k^{+}}^{\prime} M_{k} \varepsilon_{T-k} \\
& +T^{-1} \varepsilon_{T-k}^{\prime} M_{k} \zeta_{k^{+}} \beta(k+1, \infty)^{\prime}+T^{-1} \varepsilon_{T-k}^{\prime} \varepsilon_{T-k}-T^{-1} \varepsilon_{T-k}^{\prime}\left(\zeta_{k}\left(\zeta_{k}^{\prime} \zeta_{k}\right)^{-1} \zeta_{k}^{\prime}\right) \varepsilon_{T-k} \\
& =(I)+(I I)+(I I I)+(I V)+(V)=T^{-1} \varepsilon_{T-k}^{\prime} \varepsilon_{T-k}+o_{p}(1) .
\end{aligned}
$$

That is, we need to find out a condition on the growth rate of the lag order $\mathrm{k}$ to ensure that the first term (I), the second term (II), the third term (III) and the fifth term $(\mathrm{V})$ of $(\mathrm{A} .8)$ converge to zero when $-0.5<d^{*}<0.5, d^{*} \neq 0$.

We first investigate the asymptotic properties of (A.8) for the case of $-0.5<d<$ 0 . The first term (I) on the right hand side of (A.8) can be written as

$$
\begin{aligned}
& (I)=T^{-1}\left(\beta(k+1, \infty) \zeta_{k^{+}}^{\prime} M_{k} \zeta_{k^{+}} \beta(k+1, \infty)^{\prime}\right) \\
& =T^{-1}\left\{\beta(k+1, \infty) \zeta_{k^{+}}^{\prime} I \zeta_{k^{+}} \beta(k+1, \infty)^{\prime}\right\} \\
& -T^{-1}\left\{\beta(k+1, \infty) \zeta_{k^{+}}^{\prime} \zeta_{k}\left(\zeta_{k}^{\prime} \zeta_{k}\right)^{-1} \zeta_{k}^{\prime} \zeta_{k^{+}} \beta(k+1, \infty)^{\prime}\right\}=A-B,
\end{aligned}
$$

where $A$ and $B$ denote the first and second term of the righthand side of (A.9), respectively. It can be shown that

$$
\begin{aligned}
& \left.\|A\|^{2}=\| T^{-1} \beta(k+1, \infty) \zeta_{k^{+}}^{\prime} I \zeta_{k^{+}} \beta(k+1, \infty)^{\prime}\right\} \|^{2}=O_{p}\left(k^{-4 d^{*}-2} T^{4 d^{*}}\right) \\
& \text { and }\|A\|=O_{p}\left(k^{-2 d^{*}-1} T^{2 d^{*}}\right) .
\end{aligned}
$$


To show (A.10), we note that for any conformable matrix $A^{*}$ and $B^{*}$,

$$
\left\|A^{*} B^{*}\right\|^{2} \leq\left\|A^{*}\right\|^{2}\left\|B^{*}\right\|^{2}
$$

$$
\begin{aligned}
& \text { therefore } \left.\|A\|^{2}=\| T^{-1} \beta(k+1, \infty) \zeta_{k^{+}}^{\prime} I \zeta_{k^{+}} \beta(k+1, \infty)^{\prime}\right\} \|^{2} \\
& \left.\leq T^{-2}\left\|\beta(k+1, \infty) \zeta_{k^{+}}^{\prime}\right\|^{2} \| \zeta_{k^{+}} \beta(k+1, \infty)^{\prime}\right\} \|^{2} \\
& =T^{-2}\left(\sum_{t=k+1}^{T}\left(\sum_{j=k+1}^{\infty} \beta_{j} \eta_{t-j}\right)^{2}\right)^{2}=O_{p}\left(k^{-4 d^{*}-2} T^{4 d^{*}}\right) .
\end{aligned}
$$

We note that

$$
\begin{aligned}
& \mathbf{E}\left(\sum_{j=k+1}^{\infty} \beta_{j} \eta_{t-j}\right)^{2}=\sum_{j=k+1}^{\infty} \beta_{j}^{2} \mathbf{E}\left(\eta_{t-j}^{2}\right)+\sum_{j=k+1}^{\infty} \sum_{v=j+1}^{\infty} \beta_{j} \beta_{v} \mathbf{E}\left(\eta_{t-v, i} \eta_{t-j, i}\right) \\
& \leq \sum_{j=k+1}^{\infty} \beta_{j}^{2} \gamma_{0}+\sum_{j=k+1}^{\infty} \sum_{v=j+1}^{\infty} \beta_{j} \beta_{v}\left|\gamma_{v-j}\right| .
\end{aligned}
$$

Since Lemma 1 indicates that the complete time series can be represented by another long memory process with the memory parameter $d^{*}$, we note that $\beta_{j}^{2} \sim$ $C^{2} j^{-2 d^{*}-2}, \mathrm{C}$ and $C_{2}$ being finite constants. Following the proof of Hosking (1996, p.277) and letting $x=\frac{s}{m}$, we can show that (A.12) is bounded by

$$
\begin{aligned}
& \sum_{j=k+1}^{\infty} \beta_{j}^{2} \gamma_{0}+\left|C_{2}\right| \sum_{j=k+1}^{\infty} \sum_{v=j+1}^{\infty}\left|\beta_{j}\right|\left|\beta_{v}\right||(v-j)|^{2 d^{*}-1} \\
& \leq O_{p}\left(k^{-2 d^{*}-1}\right)+k^{-d^{*}-1} \sum_{j=k+1}^{\infty} \sum_{v=j+1}^{\infty}|(v-j)|^{2 d^{*}-1} v^{-d^{*}-1} \\
& =O_{p}\left(k^{-2 d^{*}-1}\right)+k^{-d^{*}-1} \sum_{j=k+1}^{\infty} \sum_{s=1}^{\infty} s^{2 d^{*}-1}(s+j)^{-d^{*}-1} \\
& \leq O_{p}\left(k^{-2 d^{*}-1}\right)+k^{-2 d^{*}-1} \sum_{s=1}^{\infty} s^{2 d^{*}-1} \\
& \sim O_{p}\left(k^{-2 d^{*}-1}\right)+k^{-2 d^{*}-1} T^{2 d^{*}} \int_{0}^{1} x^{2 d^{*}-1} d x \text { as } T \rightarrow \infty \\
& =O_{p}\left(k^{-2 d^{*}-1}\right),
\end{aligned}
$$

where $\gamma_{\tau}=E\left(\eta_{t} \eta_{t+\tau}\right) \sim C_{2} \tau^{2 d^{*}-1}, C_{2}$ being a finite constant.

It follows that

$$
\sum_{j=k+1}^{\infty} \beta_{j} \eta_{t-j}=O_{p}\left(k^{-d^{*}-0.5}\right) .
$$


Similarly, we can show that

$$
\|B\| \leq\left\|T^{-1} \beta(k+1, \infty) \zeta_{k^{+}}^{\prime}\right\|\left\|\zeta_{k}\left(\zeta_{k}^{\prime} \zeta_{k}\right)^{-1} \zeta_{k}^{\prime} \zeta_{k^{+}} \beta(k+1, \infty)^{\prime}\right\|,
$$

where by (A.13) and (A.14),

$$
\begin{aligned}
& \left\|T^{-1} \beta(k+1, \infty) \zeta_{k^{+}}^{\prime}\right\|^{2}=T^{-2} \sum_{t=k+1}^{T}\left(\sum_{j=k+1}^{\infty} \beta_{j} \eta_{t-j}\right)^{2}=O_{p}\left(k^{-2 d^{*}-1} T^{-1}\right) \\
& \quad \text { and }\left\|T^{-1} \beta(k+1, \infty) \zeta_{k^{+}}^{\prime}\right\|=O_{p}\left(k^{-d^{*}-0.5} T^{-0.5}\right) \\
& \left\|\zeta_{k}\left(\zeta_{k}^{\prime} \zeta_{k}\right)^{-1} \zeta_{k}^{\prime} \zeta_{k^{+}} \beta(k+1, \infty)^{\prime}\right\|^{2} \\
& =\operatorname{tr}\left(\beta(k+1, \infty) \zeta_{k^{+}}^{\prime} \zeta_{k}\left(\zeta_{k}^{\prime} \zeta_{k}\right)^{-1 \prime} \zeta_{k} \zeta_{k}\left(\zeta_{k}^{\prime} \zeta_{k}\right)^{-1} \zeta_{k}^{\prime} \zeta_{k^{+}} \beta(k+1, \infty)^{\prime}\right) \\
& =\sum_{t=k+1}^{T}\left(\sum_{j=k+1}^{\infty} \beta_{j} \eta_{t-j}\right)^{2}=O_{p}\left(T^{1} k^{-2 d^{*}-1}\right) \\
& \quad \text { and }\left\|\zeta_{k}\left(\zeta_{k}^{\prime} \zeta_{k}\right)^{-1} \zeta_{k}^{\prime} \zeta_{k^{+}} \beta(k+1, \infty)^{\prime}\right\|=O_{p}\left(T^{0.5} k^{-d^{*}-0.5}\right)
\end{aligned}
$$

Thus, by (A.16) and (A.18),

$$
B=O_{p}\left(k^{-2 d^{*}-1}\right) .
$$

Furthermore, (A.11) and (A.19) imply that

$$
(I)=T^{-1}\left(\beta(k+1, \infty) \zeta_{k^{+}}^{\prime} M_{k} \zeta_{k^{+}} \beta(k+1, \infty)^{\prime}\right)=O_{p}\left(k^{-2 d^{*}-1}\right) .
$$

We express the second term (II) on the right side of (A.8) as

$$
\begin{aligned}
(I I)= & T^{-1} \beta(k+1, \infty) \zeta_{k^{+}}^{\prime} M_{k} \varepsilon_{T-k}=T^{-1} \beta(k+1, \infty) \zeta_{k^{+}}^{\prime} \varepsilon_{T-k} \\
& -T^{-1} \beta(k+1, \infty) \zeta_{k^{+}} \zeta_{k}\left(\zeta_{k}^{\prime} \zeta_{k}\right)^{-1} \zeta_{k}^{\prime} \varepsilon_{T-k}=C-D,
\end{aligned}
$$

where $C, D$ denote the first and second term of the righthand side of (A.21), respectively. Noting that

$$
\begin{aligned}
& \left\|T^{-1} \beta(k+1, \infty) \zeta_{k^{+}}^{\prime} \varepsilon_{T-k}\right\|^{2}=T^{-2}\left\|\beta(k+1, \infty) \zeta_{k^{+}}^{\prime}\right\|^{2}\left\|\varepsilon_{T-k}\right\|^{2} \\
& =O_{p}\left(T^{-1} k^{-2 d^{*}-1}\right)
\end{aligned}
$$

it follows that

$$
C=O_{p}\left(T^{-0.5} k^{-d^{*}-0.5}\right) .
$$

Similarly, by (A.21), we can show that

$$
\begin{aligned}
& \|D\|^{2}=\left\|T^{-1} \beta(k+1, \infty) \zeta_{k^{+}} \zeta_{k}\left(\zeta_{k}^{\prime} \zeta_{k}\right)^{-1} \zeta_{k}^{\prime} \varepsilon_{T-k}\right\|^{2} \\
& =T^{-2} \operatorname{tr}\left(\varepsilon_{T-k} \zeta_{k}\left(\zeta_{k}^{\prime} \zeta_{k}\right)^{\prime-1} \zeta_{k}^{\prime} \zeta_{k^{+}}^{\prime} \beta(k+1, \infty)^{\prime} \beta(k+1, \infty) \zeta_{k^{+}} \zeta_{k}\left(\zeta_{k}^{\prime} \zeta_{k}\right)^{-1} \zeta_{k}^{\prime} \varepsilon_{T-k}\right) \\
& =O_{p}\left(T^{-1} k^{-2 d^{*}-1}\right) .
\end{aligned}
$$




$$
\text { Thus, }(I I)=O_{p}\left(T^{-0.5} k^{-d^{*}-0.5}\right) \text {. }
$$

Likewise, the third term (III) on the right side of (A.7),

$$
(I I I)=\varepsilon_{T-k}^{\prime} M_{k} \zeta_{k^{+}} \beta(k+1, \infty)=O_{p}\left(T^{-0.5} k^{-d^{*}-0.5}\right) .
$$

To calculate the asymptotic properties of the fifth term $(\mathrm{V})$ on the right side of (A.8), we further note that

$$
\begin{aligned}
\widehat{\Omega}_{k} & =\frac{\zeta_{k}^{\prime} \zeta_{k}}{T-k} \\
& =\left[\begin{array}{cccc}
\frac{1}{T-k} \sum_{i=k+1}^{T} \eta_{i-1}^{2} & \frac{1}{T-k} \sum_{i=k+1}^{T} \eta_{i-1} \eta_{i-2} & \cdots & \frac{1}{T-k} \sum_{i=k+1}^{T} y_{i-1} y_{i-k} \\
\ldots & \cdots & \cdots & \ldots \\
\vdots & \vdots & \vdots & \vdots \\
\frac{1}{T-k} \sum_{i=k+1}^{T} y_{i-k} y_{i-1} & \cdots & \ldots & \frac{1}{T-k} \sum_{i=k+1}^{T} \eta_{i-k}^{2}
\end{array}\right]
\end{aligned}
$$

and

$$
\Omega_{k}=\mathbf{E}\left(\zeta_{k}^{\prime} \zeta_{k}\right)=\left[\begin{array}{cccc}
\mathbf{E} \eta_{i-1}^{2} & \mathbf{E} \eta_{i-1} \eta_{i-2} & \cdots & \mathbf{E} \eta_{i-1} \eta_{i-k} \\
\cdots & \cdots & \cdots & \cdots \\
\vdots & \vdots & \vdots & \vdots \\
\mathbf{E} \eta_{i-k} \eta_{i-1} & \cdots & \cdots & \mathbf{E} \eta_{i-k}^{2}
\end{array}\right]
$$

By Theorem 3 of Hosking (1996) and Theorem 4.1 of Poskitt (2007), we can show that

$$
\begin{gathered}
\left\|\widehat{\Omega}_{k}-\Omega_{k}\right\|=O_{p}\left(k(\log T / T)^{0.5-d^{*}}\right), \quad d^{*} \in(-0.5,0.5), d^{*} \neq 0, \quad \text { (A.26) } \\
\text { Thus, }\left\|\widehat{\Omega}_{k}^{-1}\right\| \leq\left\|\widehat{\Omega}_{k}^{-1}-\Omega_{k}^{-1}\right\|+\left\|\Omega_{k}^{-1}\right\|=\left\|\widehat{\Omega}_{k}^{-1}\left(\Omega_{k}-\widehat{\Omega}_{k}\right) \Omega_{k}^{-1}\right\|+\left\|\Omega_{k}^{-1}\right\| \\
=\left\|\widehat{\Omega}_{k}^{-1}\right\|\left\|\left(\Omega_{k}-\widehat{\Omega}_{k}\right)\right\|\left\|\Omega_{k}^{-1}\right\|+\left\|\Omega_{k}^{-1}\right\| \\
\text { and }\left\|\Omega_{k}^{-1}\right\|<\infty,
\end{gathered}
$$

because $\Omega_{k}$ is a nonsingular matrix and by Lemma 5.7 of Poskitt (2007) and Proposition 4.5.3. of Brockwell and Davis (1991). Hence, by (A.26),

$$
\left\|\widehat{\Omega}_{k}^{-1}\right\| \leq \frac{\left\|\Omega_{k}^{-1}\right\|}{\left(1-\left\|\left(\Omega_{k}-\widehat{\Omega}_{k}\right)\right\|\left\|\Omega_{k}^{-1}\right\|\right)}=O_{p}\left(k^{-1}(\log T / T)^{d^{*}-0.5}\right) .
$$

Thus, by (A.27) the fifth term (V) can be written

$$
\begin{aligned}
& \left\|T^{-1} \varepsilon_{T-k}^{\prime} \zeta_{k}\left(\zeta_{k}^{\prime} \zeta_{k}\right)^{-1} \zeta_{k}^{\prime} \varepsilon_{T-k}\right\|^{2}=\left\|T^{-1} \varepsilon_{T-k}^{\prime}\left(\zeta_{k}\left(\frac{\zeta_{k}^{\prime} \zeta_{k}}{T-k}\right)^{-1} \zeta_{k}^{\prime}\right)^{\prime} \frac{1}{T-k} \varepsilon_{T-k}\right\|^{2} \\
& \leq\left\|T^{-1} \varepsilon_{T-k} \zeta_{k}\right\|^{2}\left\|\left(\frac{\zeta_{k}^{\prime} \zeta_{k}}{T}\right)^{-1}\right\|^{2}\left\|\frac{1}{T} \varepsilon_{T} \zeta_{k}^{\prime}\right\|^{2} \\
& =O_{p}\left(k T^{-1}\right) O_{p}\left(k^{-2}(\log T / T)^{-1+2 d^{*}}\right) O_{p}\left(k T^{-1}\right) \\
& =O_{p}\left((\log T)^{-1+2 d^{*}} T^{-2 d^{*}-1}\right) .
\end{aligned}
$$


Thus by (A.28), we note that

$$
\begin{aligned}
& \frac{1}{T} \varepsilon_{T-k}^{\prime} M_{k} \varepsilon_{T-k}=\frac{1}{T} \varepsilon_{T-k}^{\prime} \varepsilon_{T-k}-\frac{1}{T} \varepsilon_{T-k}^{\prime}\left(\zeta_{k}\left(\zeta_{k}^{\prime} \zeta_{k}\right)^{-1} \zeta_{k}^{\prime} \varepsilon_{T-k}\right) \\
& =\frac{1}{T} \varepsilon_{T-k}^{\prime} \varepsilon_{T-k}-O_{p}\left((\log T)^{d^{*}-0.5} T^{-d^{*}-0.5}\right) .
\end{aligned}
$$

For the case of $0<d<0.5$, we reprove (A.12) and (A.13) as

$$
\mathbf{E}\left(\sum_{j=k+1}^{\infty} \beta_{j} \eta_{t-j}\right)^{2}=O_{p}\left(k^{-2 d^{*}-1} T^{2 d^{*}}\right)
$$

Therefore, by (A.30) and following the similar proof procedure for the case of $-0.5<$ $d<0$, when $0<d<0.5$,

$$
\begin{gathered}
(I)=T^{-1}\left(\beta(k+1, \infty) \zeta_{k^{+}}^{\prime} M_{k} \zeta_{k^{+}} \beta(k+1, \infty)^{\prime}\right)=O_{p}\left(k^{-2 d^{*}-1} T^{2 d^{*}}\right), \\
(I I)=T^{-1} \beta(k+1, \infty) \zeta_{k^{+}}^{\prime} M_{k} \varepsilon_{T-k}=O_{p}\left(k^{-d^{*}-0.5} T^{d^{*}-0.5}\right) \\
(I I I)=\varepsilon_{T-k}^{\prime} M_{k} \zeta_{k^{+}} \beta(k+1, \infty)=O_{p}\left(T^{d^{*}-0.5} k^{-d^{*}-0.5}\right) .
\end{gathered}
$$

Therefore, when we restrict $\mathrm{k}$ on the growth rate of $T^{r}, r>2 d^{*} /\left(1+2 d^{*}\right)$, i.e. $k=O\left(T^{r}\right), r>2 d^{*} /\left(1+2 d^{*}\right),(\mathrm{A} .20),(\mathrm{A} .24),(\mathrm{A} .25),(\mathrm{A} .28),(\mathrm{A} .31),(\mathrm{A} .32)$ and (A.33) converge to zero. It follows that (I), (II), (III) and (V) converge to zero when $-0.5<d^{*}<0.5$. Hence, when $k=O\left(T^{r}\right), r>2 d^{*} /\left(1+2 d^{*}\right)$ and $-0.5<d^{*}<0.5$,

$$
\widehat{Q}=T^{-1} \varepsilon_{T-k}^{\prime} \varepsilon_{T-k}+o_{p}(1) .
$$

Furthermore, by (A.16), (A.27), (A.28) and (A.30), we note that when $-0.5<$ $d^{*}<0.5$,

$$
\begin{aligned}
& \|\beta(k)-\beta\| \leq\left\|\zeta_{k}\left(\zeta_{k}^{\prime} \zeta_{k}\right)^{-1} \zeta_{k}^{\prime} \zeta_{k^{+}} \beta(k+1, \infty)^{\prime}\right\| \\
& +\left\|\left(\zeta_{k}^{\prime} \zeta_{k}\right)^{-1} \zeta_{k}^{\prime} \varepsilon_{T-k}\right\|=O_{p}\left(k^{-1 / 2}(\log T)^{d^{*}-0.5} T^{-d^{*}}\right) .
\end{aligned}
$$

\section{A.3. Proof of Theorem 2}

By Lemma 1, Brockwell and Davis (1991) and Poskitt (2007), we denote the $h-$ step best linear prediciton of $\eta_{t}$ as

$$
\eta_{t+h}^{*}=\mu^{*}+\sum_{j=h}^{\infty} \psi_{j} e_{t+h-j}, \quad \text { where } \quad \psi \sim j^{d^{*}-1}
$$

$$
\text { or } \quad \eta_{t+h}^{*}=(1-L)^{d^{*}} \mu^{*}+\sum_{j=1}^{k} \beta_{j k} \eta_{t+h-j}, \quad \text { where } \quad \pi \sim j^{-d^{*}-1}
$$


Thus, by (7), Theorem 1, (A.36) and (A.37), we note that the $h$ - step ahead mean squared error of the forecst $\widehat{\eta}_{T+h}$ based on the $A R(k)$ approximation model is

$$
\begin{aligned}
& M S E(h)^{A R}=E\left(\eta_{T+h}-\widehat{\eta}_{T+h}\right)^{2}=E\left(\eta_{T+h}-\eta_{T+h}^{*}+\eta_{T+h}^{*}-\widehat{\eta}_{T+h}\right)^{2} \\
& =E\left(\eta_{T+h}-\eta_{T+h}^{*}\right)^{2}+E\left(\eta_{T+h}^{*}-\widehat{\eta}_{T+h}\right)^{2}+2 E\left(\left(\eta_{T+h}-\eta_{T+h}^{*}\right)\left(\eta_{T+h}^{*}-\widehat{\eta}_{T+h}\right)\right)=\sigma^{2} \sum_{j=0}^{h-1} \psi_{j}^{2} \\
& +(1-L)^{d^{*}} \kappa^{2}\left(\mu_{1}-\mu_{2}\right)^{2}+O_{p}\left(k^{-1} T^{-2 d^{*}}(\log T)^{2 d^{*}-1}\right)+O_{p}\left(k^{-1 / 2} T^{-d^{*}}(\log T)^{d^{*}-0.5}\right) . \quad
\end{aligned}
$$

Because $\pi_{i, j}=\frac{\Gamma\left(j-d_{i}\right)}{\Gamma(j+1) \Gamma\left(d_{i}\right)}, i=1,2$ and $\pi_{j}^{*}=\frac{\Gamma\left(j-d^{*}\right)}{\Gamma(j+1) \Gamma\left(d^{*}\right)}$, by the Stirling's formula, we note that as $j \rightarrow \infty$,

$$
\pi_{i, j} \sim j^{-d_{i}-1} / \Gamma\left(-d_{i}\right), i=1,2, \quad \text { and } \quad \pi_{j}^{*} \sim j^{-d^{*}-1} / \Gamma\left(-d^{*}\right),
$$

thus

$$
\begin{aligned}
& \tilde{\pi}_{j}^{*}-\pi_{j}^{*}=\frac{\Gamma\left(j-\widetilde{d}^{*}\right)}{\Gamma(j+1) \Gamma\left(\widetilde{d}^{*}\right)}-\frac{\Gamma\left(j-d^{*}\right)}{\Gamma(j+1) \Gamma\left(d^{*}\right)} \\
& \sim j^{-\widetilde{d}^{*}-1} / \Gamma\left(-\widetilde{d}^{*}\right)-j^{-d^{*}-1} / \Gamma\left(-d^{*}\right) .
\end{aligned}
$$

Furthermore, it is well known that $T^{1 / 2}\left(\widetilde{d}_{i}-d_{i}\right) \Rightarrow N\left(0,6 / \pi^{2}\right), i=1,2$. (See Baillie 1996). Because $d^{*} \leq \max \left\{d_{1}, d_{2}\right\}$, for ease of notation, we suppose that $d^{*}=d_{2}$ and $\tilde{\pi}_{j}^{*}=\widetilde{\pi}_{2, j}$. By Slutzky Theorem, $\widetilde{\pi}_{2, j}$ converges to $\pi_{2, j}$. Then, by Taylor's Theorem, we note that

$$
\tilde{\pi}_{2, j}-\pi_{2, j} \simeq c j^{-d_{2}-1} \ln j\left(\widetilde{d}_{2}-d_{2}\right)=O_{p}\left(j^{-d_{2}-1} \ln j T^{-1 / 2}\right) O_{p}\left(T^{-1}\right) .
$$

and letting $i-j=s$ and $T-j=u$,

$$
\begin{aligned}
& \mathbf{E}\left(\sum_{j=1}^{T}\left(\widetilde{\pi}_{2, j}-\pi_{2, j}\right) \eta_{T+h-j}\right)^{2} \\
& =\sum_{j=1}^{T}\left(\widetilde{\pi}_{2, j}-\pi_{2, j}\right)^{2} E\left(\eta_{T+h-j}\right)^{2}+\sum_{j=1}^{T-1} \sum_{i=j+1}^{T}\left(\widetilde{\pi}_{2, j}-\pi_{2, j}\right)\left(\widetilde{\pi}_{2, i}-\pi_{2, i}\right) \gamma_{i-j} \\
& \cong\left(\widetilde{d}_{2}-d_{2}\right)^{2} \sigma_{\eta}^{2} \sum_{j=1}^{T}\left(j^{-d_{2}-1} \ln j\right)^{2}+\sum_{j=1}^{T-1} \sum_{i=j+1}^{T}\left(\widetilde{d}_{2}-d_{2}\right)^{2}\left(j^{-d_{2}-1} \ln j\right)\left(i^{-d_{2}-1} \ln i\right)(i-j)^{2 d_{2}-1} \\
& =C\left(6 / \pi^{2}\right) \sigma_{\eta}^{2} \frac{(\ln T)^{2}}{T}+\frac{C\left(6 / \pi^{2}\right)}{T} \sum_{j=1}^{T-1} j^{-d_{2}-1} \ln j\left((T-j)^{d_{2}-1} \ln (T-j)\right) \\
& =C\left(6 / \pi^{2}\right) \sigma_{\eta}^{2} \frac{(\ln T)^{2}}{T}+O_{p}\left(\frac{(\ln T)^{2}}{T}\right) .
\end{aligned}
$$


Thus, by (A.37), (A.38) and (A.42), the $h$-step mean squared error of the NVNO forecast $\widetilde{\eta}_{T+h}$ given by (8) is

$$
\begin{aligned}
& M S E(h)^{N V N O}=E\left(\eta_{T+h}-\widetilde{\eta}_{T+h}\right)^{2}=E\left(\eta_{T+h}-\eta_{T+h}^{*}+\eta_{T+h}^{*}-\widetilde{\eta}_{T+h}\right)^{2} \\
& =\sigma^{2} \sum_{j=0}^{h-1} \psi_{j}^{2}+(1-L)^{d^{*}} \kappa^{2}\left(\mu_{1}-\mu_{2}\right)^{2}+C\left(6 / \pi^{2}\right) \sigma_{\eta}^{2} \frac{(\ln T)^{2}}{T}+O_{p}\left(\frac{(\ln T)^{2}}{T}\right) \\
& +C\left(6 / \pi^{2}\right)^{1 / 2} \sigma_{\eta} \frac{\ln T}{T^{1 / 2}}+O_{p}\left(\frac{\ln T}{T^{1 / 2}}\right),
\end{aligned}
$$

and that of the post-break forecast $\widehat{\eta}_{T+h}^{*}$ given by (10) is

$$
\begin{aligned}
& M S E(h)^{P B}=E\left(\eta_{T+h}-\widehat{\eta}_{T+h}^{*}\right)^{2}=E\left(\eta_{T+h}-\eta_{T+h}^{*}+\eta_{T+h}^{*}-\widehat{\eta}_{T+h}^{*}\right)^{2} \\
& =\sigma^{2} \sum_{j=0}^{h-1} \psi_{j}^{2}+C\left(6 / \pi^{2}\right) \sigma_{\eta}^{2} \frac{\left(\ln T_{2}\right)^{2}}{T_{2}}+O_{p}\left(\frac{\left(\ln T_{2}\right)^{2}}{T_{2}}\right) \\
& +C\left(6 / \pi^{2}\right)^{1 / 2} \sigma_{\eta} \frac{\ln T_{2}}{T_{2}^{1 / 2}}+O_{p}\left(\frac{\ln T_{2}}{T_{2}^{1 / 2}}\right) .
\end{aligned}
$$

Furthermore, to prove the $h$-step mean squared error of the NV method, We first obtain that as $\pi_{j}^{*}=\pi_{2, j}$,

$$
\begin{aligned}
& \mathbf{E}\left(\sum_{j=T_{2}+1}^{T}\left(\tilde{\pi}_{j}^{*}-\pi_{2, j}\right) \eta_{T+h-j}\right)^{2} \\
& \leq C\left(6 / \pi^{2}\right) \sigma_{y}^{2} \frac{\left(\ln T_{2}\right)^{2}}{T_{2}}+O_{p}\left(\frac{\left(\ln T_{2}\right)^{2}}{T_{2}}\right)=O_{p}\left(\left(\ln T_{2}\right)^{2} T_{2}^{-1}\right) .
\end{aligned}
$$

By Hosking (1981), we note that $(1-L)^{d_{3}}=C$ as $d_{3}>0$ and $(1-L)^{d_{3}}=$ $O\left(T^{-d_{3}}\right)$ as $d_{3}<0$, where $d_{3}=d_{2}-d_{1}$. Then following the analysis of (A.42), we can get

$$
\begin{aligned}
& E\left(\sum_{j=T_{2}+1}^{T}(1-L)^{d_{3}} \pi_{2, j} \mu_{1}\right)^{2} \leq \mu_{1}^{2}(1-L)^{2 d_{3}} \mathbf{E}\left(\sum_{j=T_{2}+1}^{T} \pi_{2, j}\right)^{2} \\
& \leq \mu_{2}^{2}\left(\sum_{j=T_{2}+1}^{T} \mathbf{E}\left(j^{-d_{2}-1}\right)^{2}+\sum_{j=T_{2}+1}^{T} \sum_{i=j+1}^{T-1} \mathbf{E}\left(j^{-d_{2}-1} i^{-d_{2}-1}\right)\right) \\
& =C \mu_{1}^{2} O\left(T_{2}^{-2 d_{3}-2 d_{2}-1}\right) \\
& \text { and } \quad E\left(\sum_{j=T_{2}+1}^{T} \pi_{2, j} \mu_{2}\right)^{2} \leq \mu_{2}^{2} \mathbf{E}\left(\sum_{j=T_{2}+1}^{T} \pi_{2, j}\right)^{2}=C \mu_{1}^{2} O\left(T_{2}^{-2 d_{2}-1}\right) .
\end{aligned}
$$


Following the analysis of (A.42) and (A.46),

$$
\mathbf{E}\left(\sum_{j=T_{2}+1}^{T}\left(\tilde{\pi}_{j}^{*}-(1-L)^{d_{3}} \pi_{2, j}\right) \eta_{T+h-j}\right)^{2}=O_{p}\left((\ln T)^{2} T^{-1}\right) .
$$

Moreover, by Baillie (1996), we note that $\widetilde{\mu}_{i}-\mu_{i}=O_{p}\left(T^{-1 / 2}\right), i=1,2(A .49)$. Because $\mu^{*} \neq \mu_{2}$, when $d^{*}=d_{2}$, we note that as $T \rightarrow \infty$,

$$
\begin{aligned}
& \mathbf{E}\left((1-L)^{d_{2}} \mu_{2}-(1-L)^{\widetilde{d}^{*}} \widetilde{\mu}^{*}\right)^{2} \\
& =\mathbf{E}\left((1-L)^{d_{2}} \mu_{2}-(1-L)^{\tilde{d}^{*}}\left(\kappa \widetilde{\mu}_{1}+(1-\kappa) \widetilde{\mu}_{2}\right)\right)^{2} \\
& =\kappa^{2}(1-L)^{2 d_{2}}\left(\mu_{2}-\mu_{1}\right)^{2} .
\end{aligned}
$$

Then by (A.36), (A.42), (A.46), (A.47), (A.48), (A.49) and (A.50), when $a=$ $1,(1-a)=0$ (i.e., type II error), the mean square error of $\widetilde{\eta}_{T+h}^{I I}$ is

$$
\begin{aligned}
& \operatorname{MSE}(h)^{N A^{I I}}=\sigma^{2} \sum_{j=0}^{h-1} \psi_{j}^{2}+E\left(\left((1-L)^{d_{2}} \mu_{2}-(1-L)^{d^{*}} \widetilde{\mu}^{*}-\sum_{j=1}^{T_{2}}\left(\widetilde{\pi}_{j}^{*}-\pi_{2, j}\right) \eta_{T+1-j}\right)\right. \\
& \left.-\sum_{j=T_{2}+1}^{T}\left(\widetilde{\pi}_{j}^{*}-(1-L)^{d_{3}} \pi_{2, j}\right) \eta_{T+1-j}+\sum_{j=T_{2}+1}^{T}(1-L)^{d_{3}} \pi_{2, j} \mu_{1}-\sum_{j=T_{2}+1}^{T} \pi_{2, j} \mu_{2}\right)^{2} \\
& =\sigma^{2} \sum_{j=0}^{h-1} \psi_{j}^{2}+O_{p}\left(\frac{\left(\ln T_{2}\right)^{2}}{T_{2}}\right)+\kappa^{2}(1-L)^{d_{2}}\left(\mu_{2}-\mu_{1}\right)^{2}+O_{p}\left(T_{2}^{-2 d_{2}-2 d_{3}-1}\right) \\
& +O_{p}\left(\frac{\left(\ln T_{2}\right)}{T_{2}^{1 / 2}}\right) .
\end{aligned}
$$

Moreover, when $a=0,(1-a)=1$ (i.e., finding a break), then we use a naive ARFIMA model with the pre-and post break estimated parameters $\widetilde{d}_{1}$ and $\widetilde{d}_{2}$ to compute a forecast. Using (A.36), (A.42), (A.46), (A.47), (A.48), (A.50), the mean square error of $\widetilde{\eta}_{T+h}^{I I}$ is

$$
\begin{aligned}
& M S E(h)^{N A^{I}}=\sigma^{2} \sum_{j=0}^{h-1} \psi_{j}^{2}+E\left(\left((1-L)^{d_{2}} \mu_{2}-(1-L)^{\widetilde{d}_{2}} \widetilde{\mu}_{2}-\sum_{j=1}^{T_{2}}\left(\widetilde{\pi}_{2, j}-\pi_{2, j}\right) \eta_{T+1-j}\right)\right. \\
& \left.-\sum_{j=T_{2}+1}^{T}\left((1-L)^{\widetilde{d}_{3}} \widetilde{\pi}_{2, j}-(1-L)^{d_{3}} \pi_{2, j}\right) \eta_{T+1-j}\right) \\
& \left.+\sum_{j=T_{2}+1}^{T}\left((1-L)^{\widetilde{d}_{3}} \widetilde{\pi}_{2, j} \widetilde{\mu}_{1}-(1-L)^{d_{3}} \pi_{2, j} \mu_{1}\right)-\sum_{j=T_{2}+1}^{T}\left(\widetilde{\pi}_{2, j} \widetilde{\mu}_{2}-\pi_{2, j} \mu_{2}\right)\right)^{2} \\
& =\sigma^{2} \sum_{j=0}^{h-1} \psi_{j}^{2}+O_{p}\left(\frac{\left(\ln T_{2}\right)^{2}}{T_{2}}\right)+O_{p}\left(T_{2}^{-2 d_{2}-2 d_{3}-1}\right)+O_{p}\left(\frac{\left(\ln T_{2}\right)}{T_{2}^{1 / 2}}\right) .
\end{aligned}
$$


Similarly, by (A.51), when $b=1$, the mean square error of $\widehat{\eta}_{T+1}^{h}$ is

$$
\begin{aligned}
& \operatorname{MSE}(h)^{N A^{I}}=\sigma^{2} \sum_{j=0}^{h-1} \psi_{j}^{2}+E\left(\left((1-L)^{d_{2}} \mu_{2}-(1-L)^{\widetilde{d}_{2}} \widetilde{\mu}_{2}-\sum_{j=1}^{T_{2}}\left(\widetilde{\pi}_{1, j}-\pi_{2, j}\right) \eta_{T+1-j}\right)\right. \\
& \left.\left.+\sum_{j=T_{2}+1}^{T}(1-L)^{d_{3}} \pi_{2, j}\right) \eta_{T+1-j}-\sum_{j=T_{2}+1}^{T}(1-L)^{d_{3}} \pi_{2, j} \mu_{1}-\sum_{j=T_{2}+1}^{T} \pi_{2, j} \mu_{2}\right)^{2} \\
& =\sigma^{2} \sum_{j=0}^{h-1} \psi_{j}^{2}+O_{p}\left(\frac{\left(\ln T_{2}\right)^{2}}{T_{2}}\right)+O_{p}\left(T_{2}^{-2 d_{2}-2 d_{3}-1}\right)+O_{p}\left(\frac{\left(\ln T_{2}\right)}{T_{2}^{1 / 2}}\right)
\end{aligned}
$$

and when $b=0$ and $(1-b)=1$, by $(\mathrm{A} .52)$,

$$
\begin{aligned}
& M S E(h)^{N A^{I}}=\sigma^{2} \sum_{j=0}^{h-1} \psi_{j}^{2}+E\left(\left((1-L)^{d_{2}} \mu_{2}-(1-L)^{\widetilde{d}_{2}} \widetilde{\mu}_{2}-\sum_{j=1}^{T_{2}}\left(\widetilde{\pi}_{2, j}-\pi_{2, j}\right) \eta_{T+1-j}\right)\right. \\
& \left.-\sum_{j=T_{2}+1}^{T}\left((1-L)^{\widetilde{d}_{3}} \widetilde{\pi}_{2, j}-(1-L)^{d_{3}} \pi_{2, j}\right) \eta_{T+1-j}\right) \\
& \left.+\sum_{j=T_{2}+1}^{T}\left((1-L)^{\widetilde{d}_{3}} \widetilde{\pi}_{2, j} \widetilde{\mu}_{1}-(1-L)^{d_{3}} \pi_{2, j} \mu_{1}\right)-\sum_{j=T_{2}+1}^{T}\left(\widetilde{\pi}_{2, j} \widetilde{\mu}_{2}-\pi_{2, j} \mu_{2}\right)\right)^{2} \\
& =\sigma^{2} \sum_{j=0}^{h-1} \psi_{j}^{2}+O_{p}\left(\frac{\left(\ln T_{2}\right)^{2}}{T_{2}}\right)+O_{p}\left(T_{2}^{-2 d_{2}-2 d_{3}-1}\right)+O_{p}\left(\frac{\left(\ln T_{2}\right)}{T_{2}^{1 / 2}}\right) .
\end{aligned}
$$

By (A.38) and (A.43), it follows that when $k=O\left((\ln T)^{-1}(T / \ln T)^{1-2 d^{*}}\right)$, the convergence rate of the term of $O_{p}\left(\frac{(\ln T)^{2}}{T}\right)$ in equation (A.43) is slower than that of the term of $O_{p}\left(k^{-1} T^{-2 d^{*}}(\log T)^{2 d^{*}-1}\right)$ in equation (A.38), thus our AR-approximaiton based forecast method (AR) outperforms ARFIMA(d*) (NVNO) for Case I and Case II. Furthermore, by (A.38), (A.43), (A.51), (A.52), (A.53) and (A.54), we know that Theorem 2 can be established immediately.

\section{A.4. Proof of Lemma 2}

By Tiao and Tsay (1994), we note that for the adaptive forecasting, the maximum likelihood estimator (MLE) is asymptotically equivalent to the estimator obtained by minimizing the sum of squares of $h$-step-ahead forecast errors. Furthermore, by Brockwell and Davis (1991) and Poskitt (2007), we also note that the MLE and least square AR estimators are asympotitically equivalent. Thus, following the proof of Theorem 2, Lemma 2 can be established immediately. 